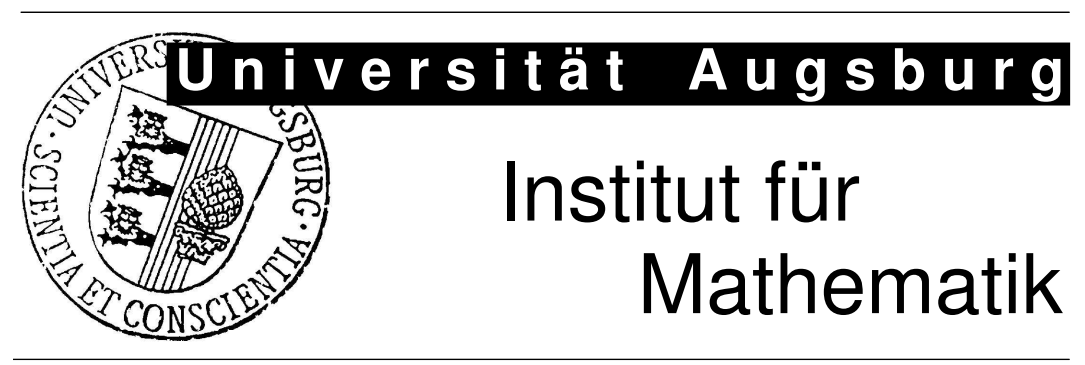

Christian Pötzsche, Martin Rasmussen

Computation of nonautonomous invariant and inertial manifolds

Preprint Nr. 041/2007 - 10. Dezember 2007

Institut für Mathematik, Universitätsstraße, D-86 135 Augsburg

http: //www.math. uni-augsburg.de/ 


\section{Impressum:}

Herausgeber:

Institut für Mathematik

Universität Augsburg

86135 Augsburg

http://www . math.uni-augsburg.de/forschung/preprint/

ViSdP:

Martin Rasmussen

Institut für Mathematik

Universität Augsburg

86135 Augsburg

Preprint: Sämtliche Rechte verbleiben den Autoren (C) 2007 


\author{
Christian Pötzsche · Martin Rasmussen
}

\title{
Computation of nonautonomous invariant and inertial manifolds
}

Received: December 3, 2007/ Revised: date

\begin{abstract}
We derive a numerical scheme to compute invariant manifolds for timevariant discrete dynamical systems, i.e., nonautonomous difference equations. Our universally applicable method is based on a truncated Lyapunov-Perron operator and computes invariant manifolds using a system of nonlinear algebraic equations which can be solved both locally using (nonsmooth) inexact Newton, and globally using continuation algorithms. Compared to other algorithms, our approach is quite flexible, since it captures time-dependent, nonsmooth, noninvertible or implicit equations and enables us to tackle the full hierarchy of strongly stable, stable and center-stable manifolds, as well as their unstable counterparts.

Our results are illustrated using a test example and are applied to a population dynamical model and the Hénon map. Finally, we discuss a linearly implicit Euler-Bubnov-Galerkin discretization of a reaction diffusion equation in order to approximate its inertial manifold.
\end{abstract}

Keywords Invariant manifold · Invariant fiber bundle · Inertial manifold · Lyapunov-Perron equation · Newton method · Continuation method · Path following $\cdot$ Bubnov-Galerkin approximation

Mathematics Subject Classification (2000) 65P99 - 37D10 - 39A11 · 37C60 • $65 \mathrm{H} 10 \cdot 65 \mathrm{~J} 15 \cdot 65 \mathrm{Q} 05$

Christian Pötzsche

Centre for Mathematical Sciences, Munich University of Technology, Boltzmannstraße 3, 85748 Garching, Germany

E-mail: christian.poetzsche@ma.tum.de

Martin Rasmussen

Department of Mathematics, University of Augsburg, 86135 Augsburg, Germany

E-mail: rasmussen@math.uni-augsburg.de 


\section{Introduction}

In qualitative studies on nonlinear dynamical systems, invariant manifolds are omnipresent and play a crucial role in a variety of ways for local as well as global questions: For instance, local stable and unstable manifolds dictate the saddlepoint behavior in the vicinity of hyperbolic solutions (or surfaces) of a system. Center manifolds are a primary tool to simplify given dynamical systems in terms of a reduction of their state space dimension (compare, e.g., the celebrated reduction principle of Pliss). Concerning a more global perspective, stable manifolds serve as separatrix between different domains of attractions and allow a classification of solutions with a specific asymptotic behavior. Systems with a gradient structure possess global attractors consisting of unstable manifolds (and equilibria). Finally, so-called inertial manifolds are global versions of the classical center-unstable manifolds and yield a global reduction principle for typically infinite-dimensional dissipative equations.

For these reasons, the computation of invariant manifolds is a highly relevant and interesting problem. However, although the existence of invariant manifolds is a well-established matter in many different settings, their analytical computation is possible only in very rare cases. Hence, one needs tools for their approximation, and at least since the 1990s, several methods have been pursued. We review some of them with a certain focus on discrete dynamical systems: Maybe the most self-evident (and theoretically for local questions relevant) approach is Taylor approximation, for which a sufficiently general framework has been introduced in [39]; moreover, Taylor expansions for large systems and related numerical issues have been discussed in $[5,12]$. For global approximations, a geometrically very intuitive approach motivated on attracting properties of unstable manifolds was suggested in [10], based on set-oriented methods like subdivision and cellmapping-continuation. Clearly, the different theoretical methods for constructing invariant manifolds imply also possibilities for their approximation. Among them is the frequently used graph transformation due to Hadamard, on which the algorithms developed in [39, Section 11], [13,6,37] (see [17] for an approach using invariant foliations) are based on, where reference [37] deals with inertial manifolds. A second method with a more functional analytical background dates back to Lyapunov and Perron, and forms the starting point for the contributions in [31] and [37] (inertial manifolds) - in each case an integral operator has to be appropriately discretized. Vaguely related to this approach is the contribution of [30], in which methods from the numerical approximation of boundary value problems are applied to compute invariant manifolds. A third theoretical method to construct invariant manifolds of differential equations is Sacker's perturbation approach based on the fact that invariant surfaces satisfy a first-order quasi-linear PDE, the so-called invariance equation. A solution scheme for such problems has been developed very successfully in [15]. However, for discrete problems, the invariance equation is a functional equation and it would be interesting to develop an analog approach for difference equations. Finally, also [16] deals with differential equations by considering whole bundles of trajectories and describing an algorithm to control them in order to approximate invariant manifolds. Various illustrative examples on this extensive area can be found in the well-written and 
interesting survey paper [24], to which we also refer for a more complete overview of the corresponding vast literature.

Being aware of these highly successful and effective methods providing a quite global picture of invariant manifolds, our motivation for the present work is threefold: First, many of the methods mentioned above deal with ordinary differential equations and in order to implement an approximation scheme for invariant manifold, one has to work with an appropriate time discretization. We deal with time-discrete problems right from the beginning and therefore our method can be applied to time-discrete models, as well as to discretized ODEs. Here, it is a typical issue that difference equations are rarely global homeomorphisms on a relevant state space, whereas numerical discretizations of ODEs are (at least for small step-sizes). Second, in many cases, the notion of a dynamical system is not general enough to model several real world phenomena, since it is often indicated to assume that the underlying rules are time-dependent. In biological processes, for instance, it is more realistic to take evolutionary adaptations into account, and sometimes it is unavoidable to consider random perturbations such as white noise or to model the control of a process by a human being. The appropriate class to treat such problems are so-called nonautonomous dynamical systems. Third, we try to reduce the smoothness assumptions on the equations under consideration. In fact, we only need their Lipschitz continuity. Such continuous, but not differentiable models occur in a variety of applications ranging from electrical circuits to linear complementary and cone-wise linear systems (see, e.g., [20] and the references therein).

Compared to the autonomous case, the literature on the approximation of invariant manifolds for nonautonomous problems is quite sparse. A local method to obtain Taylor approximations and to apply them in critical stability and bifurcation problems has been developed in [35]. However, there are two obstacles in obtaining a good, i.e., high-order Taylor approximation. First of all, the smoothness of a difference equation yields an upper bound for the differentiability of the corresponding invariant manifolds. Hence, for only Lipschitzian equations, Taylor approximations are out of question. In addition, even for $C^{\infty}$-equations, the differentiability of invariant manifolds also depends on the spectrum of the linear part in terms of spectral gap conditions, and it is possible to construct invariant manifolds which are only $C^{1}$ (cf. [32, Example 4.2]) although the equations are much smoother. Thus, a Taylor approximation is of little use in these cases.

Set-oriented methods work for only continuous right-hand sides satisfying a global Lipschitz condition on a bounded set, and have been generalized to timedependent problems in [2]. Compared to our present approach from Section 3, we got the impression that set-oriented algorithms are more robust in the sense that convergence is obtained on larger sets. Nevertheless, the computational amount is smaller for our method, and hence it appears to be faster. In addition, it directly applies to stable manifolds (without computing the inverse, which might not exist) and to implicit difference equations as well.

This paper deals with time-dependent difference equations. For readers unfamiliar with the nonautonomous theory, we have included the corresponding existence theorems for invariant manifolds (we call them invariant fiber bundles), as well as the crucial steps of their construction using the Lyapunov-Perron method. Many of the above mentioned methods to approximate invariant manifolds are 
strongly based on the autonomous invariance notion, where invariant sets are subsets of a single state space. The corresponding nonautonomous invariance concept canonically leads to the extended state space - a vector bundle of single state spaces. For that reason it is not straight forward to generalize various of the above methods to the general nonautonomous situation. However, the Lyapunov-Perron approach has a sufficiently abstract and flexible nature for two reasons:

- One can characterize the full hierarchy of invariant manifolds (or fiber bundles) including strongly stable, stable, center-stable and the corresponding unstable manifolds.

- It is successful even for time-dependent problems.

Indeed, in Section 2, invariant manifolds of nonautonomous difference equations are characterized as fixed points of the Lyapunov-Perron operator in an infinitedimensional sequence space. In Section 3, we provide an error estimate enabling us to replace this problem with a finite-dimensional system of nonlinear equations representing the truncated Lyapunov-Perron fixed point problem. This system can be solved using numerical methods for systems of nonlinear algebraic equations. We suggest and have successfully employed various Newton-like methods to compute single points on the invariant manifolds. Due to the relatively high dimension of these problems $(\approx 50-100)$, inexact Newton-methods with an iterative solution of the resulting linear problem in each Newton-step proved to be the most efficient ones. To compute whole fibers, continuation (path-following) techniques are appropriate. We naively implemented a classical continuation, as well as a pseudoarclength algorithm (cf. [1]) for that purpose. A Levenberg-Marquardt algorithm turned out to be sufficiently robust in order to use larger step-sizes as increments for the continuation parameter.

Finally, several examples are discussed in Section 4 of this paper. To demonstrate the performance of our algorithms, we investigate an autonomous planar test example where the invariant manifolds are explicitly known and, depending on the spectrum of the linear part, are of strongly stable, stable and strongly unstable type. To further illustrate our technique we take a nonautonomous version of a popular model from population dynamics (see [26]) and compare our approach to set-oriented subdivision methods from [2]. The global unstable manifold of the well-known Hénon map is approximated using pseudo-arclength continuation. The Colpitts oscillator from [28] serves as model equation with a non-smooth right-hand side. We apply an explicit Euler discretization and numerically obtain its stable and center manifold. As a concluding more complex example we consider a 2-dimensional attractive invariant manifold of a 6-dimensional difference equation. It has been obtained as Bubnov-Galerkin discretization of a nonautonomous Chafee-Infante PDE (a prototypical nonlinear heat equation in $1 d$ ), with linearly implicit Euler discretization in time, and is intended to approximate its inertial manifold (cf. [38, Chapter 8]).

\section{Difference equations and invariant fiber bundles}

Throughout the paper, $X$ is a general Banach space over the reals $(\mathbb{K}=\mathbb{R})$ or complex numbers $(\mathbb{K}=\mathbb{C})$, equipped with norm $\|\cdot\|$, and $L(X)$ the Banach algebra of bounded linear operators. All considerations hold for infinite-dimensional 
linear spaces $X$ - understandably, until it comes to explicit implementations of algorithms on a computer, where some prior spatial (Bubnov-Galerkin, finite difference, or finite element) discretization is needed.

A discrete interval $\mathbb{I}$ is the intersection of a real interval with the integers $\mathbb{Z}$. We write $[\tau, t]_{\mathbb{Z}}:=[\tau, t] \cap \mathbb{Z}$ for $\tau, t \in \mathbb{R}$, and with $T \in(0, \infty]_{\mathbb{Z}}$, we abbreviate

$$
\mathbb{I}_{\tau}^{+}(T):=\left\{\begin{array}{l}
{[\tau, \tau+T]_{\mathbb{Z}}, \quad T<\infty,} \\
{[\tau, \infty)_{\mathbb{Z}}, \quad T=\infty}
\end{array} \quad, \quad \mathbb{I}_{\tau}^{-}(T):=\left\{\begin{array}{l}
{[\tau-T, \tau]_{\mathbb{Z}}, \quad T<\infty,} \\
(-\infty, \tau]_{\mathbb{Z}}, \quad T=\infty
\end{array} .\right.\right.
$$

Since our focus is on nonautonomous problems, the classical notion of a semigroup (or dynamical system) has to be replaced by a discrete 2-parameter semigroup, i.e., a mapping $\varphi:\{(t, \tau, \xi) \in \mathbb{I} \times \mathbb{I} \times X: \tau \leq t\} \rightarrow X$ with the properties

$$
\varphi(\tau, \tau, \xi)=\xi, \quad \varphi(t, s, \varphi(s, \tau, \xi))=\varphi(t, \tau, \xi) \quad \text { for all } \tau \leq s \leq t, \xi \in X
$$

we speak of a 2-parameter group, if $\varphi$ is defined on $\mathbb{I} \times \mathbb{I} \times X$ and (1) holds for all $\tau, s, t \in \mathbb{I}$. The product $\mathbb{I} \times X$ is called extended state space and a subset $S \subseteq \mathbb{I} \times X$ is said to be a nonautonomous set with $t$-fiber $S(t):=\{x \in X:(t, x) \in S\}$. Such a nonautonomous set is called positively invariant, if $\varphi(t, \tau, S(\tau)) \subseteq S(t)$ for $\tau \leq t$ and invariant, if equality holds in the last inclusion.

Suppose from now on that the discrete interval $\mathbb{I}$ is unbounded above or below. We deal with explicit nonautonomous difference equations in semi-linear form

$$
x_{t+1}=A(t) x_{t}+F\left(t, x_{t}\right)
$$

with functions $A: \mathbb{I} \rightarrow L(X)$ and $F: \mathbb{I} \times X \rightarrow X$. For later reference, let $\varphi$ be the general solution of (2), recursively given as 2-parameter semigroup

$$
\varphi(t, \tau, \xi):=\left\{\begin{array}{cl}
\xi & \text { for } t=\tau \\
A(t-1) \varphi(t-1, \tau, \xi)+F(t-1, \varphi(t-1, \tau, \xi)) & \text { for } t>\tau
\end{array}\right.
$$

The following general pseudo-hyperbolic exponential dichotomy notion will be essential for the flexibility in our approach:

Hypothesis 1 Let $0<\alpha_{+}<\alpha_{-}, K_{+}, K_{-} \geq 1$ and $A: \mathbb{I} \rightarrow L(X)$. We assume that there exist sequences $P_{-}, P_{+}: \mathbb{I} \rightarrow L(X)$ of projections with $P_{-}(t)+P_{+}(t) \equiv I$ on $\mathbb{I}$,

$$
\begin{aligned}
& A(t+1) P_{-}(t)=P_{-}(t) A(t) \quad \text { for all } t \in \mathbb{I}, \\
& \left.A(t)\right|_{R\left(P_{-}(t)\right)}: R\left(P_{-}(t)\right) \rightarrow R\left(P_{-}(t+1)\right) \quad \text { is invertible for all } t \in \mathbb{I} \text {, } \\
& \left\|\Phi(t, s) P_{+}(s)\right\| \leq K_{+} \alpha_{+}^{t-s} \text { for all } s \leq t, \\
& \left\|\bar{\Phi}(t, s) P_{-}(s)\right\| \leq K_{-} \alpha_{-}^{t-s} \text { for all } t \leq s,
\end{aligned}
$$

where $\Phi(t, s):=\prod_{r=s}^{t-1} A(r)$ and $\bar{\Phi}(s, t):=\left.\prod_{r=s}^{t-1} A(r)\right|_{R\left(P_{-}(r)\right)} ^{-1}$ for $s \leq t$.

Remark 1 In the autonomous case (i.e., $A(t) \equiv A_{0}$ is constant), the linearized system (2) admits an exponential dichotomy for growth rates $\alpha_{+}<\alpha_{-}$, if the modulus of each spectral point of $A_{0}$ does not lie in $\left[\alpha_{+}, \alpha_{-}\right]$. Similarly, if $A$ is $\omega$-periodic, one has to consider spectral points of the monodromy operator $\Phi(\omega, 0)$. 
Because of the regularity condition (3), we know in the numerically approachable case $d:=\operatorname{dim} X<\infty$ that the ranks of the projection $P_{ \pm}(t), t \in \mathbb{I}$, are constant, and we can define

$$
d_{ \pm}:=\operatorname{dim} R\left(P_{ \pm}(t)\right) \quad \text { for all } t \in \mathbb{I} .
$$

For the sake of a compact and convenient notation it is advantageous to introduce the Green's function for $x_{t+1}=A(t) x_{t}$, given by

$$
G(t, \tau):=\left\{\begin{array}{cc}
-\bar{\Phi}(t, \tau) P_{-}(\tau) & \text { for } t<\tau \\
\Phi(t, \tau) P_{+}(\tau) & \text { for } t \geq \tau
\end{array}\right.
$$

Having this at hand, we can establish our abstract functional analytical framework. Given $\gamma>0, \tau \in \mathbb{I}$ and $T \in(0, \infty]_{\mathbb{Z}}$ such that $\tau-T \in \mathbb{I}$ or $\tau+T \in \mathbb{I}$, respectively, it is not difficult to see that the following spaces of exponentially bounded sequences

$$
X_{\tau, \gamma}^{ \pm}(T):=\left\{\phi: \mathbb{I}_{\tau}^{ \pm}(T) \rightarrow X: \sup _{t \in \mathbb{I}_{\tau}^{ \pm}(T)} \gamma^{\tau-t}\|\phi(t)\|<\infty\right\}
$$

become Banach spaces w.r.t. the respective norms

$$
\|\phi\|_{\tau, \gamma}^{ \pm}:=\sup _{t \in \mathbb{I}_{\tau}^{ \pm}(T)} \gamma^{\tau-t} \max \left\{\left\|P_{-}(t) \phi(t)\right\|,\left\|P_{+}(t) \phi(t)\right\|\right\}
$$

Note that the condition $\sup _{t \in \mathbb{I}_{\tau}^{ \pm}(T)} \gamma^{\tau-t}\|\phi(t)\|<\infty$ is always fulfilled for finite values $T<\infty$. Hence, $X_{\tau, \gamma}^{ \pm}(T)=\left\{\phi: \mathbb{I}_{\tau}^{ \pm}(T) \rightarrow X\right\} \cong X^{T+1}$ in this case.

Often an element $\phi(p) \in X_{\tau, \gamma}^{ \pm}(T)$ depends on parameters $p$, and we slightly abuse our notation by writing $\phi(t, p) \in X$ instead of the cumbersome $\phi(p)(t)$. This notational simplification will be used throughout the paper.

Hypothesis 2 Let $F: \mathbb{I} \times X \rightarrow X$ be a mapping satisfying

$$
F(t, 0) \equiv 0 \quad \text { on } \mathbb{I}
$$

and the local Lipschitz estimate

$$
\|F(t, x)-F(t, \bar{x})\| \leq L(r)\|x-\bar{x}\| \quad \text { for all } t \in \mathbb{I}, x, \bar{x} \in \bar{B}_{r}(0), r \geq 0,
$$

where the function $L:[0, \infty] \rightarrow[0, \infty]$ is nondecreasing.

Remark 2 The assumption (5) is legitimate in problems where the behavior near fixed reference solutions is in the center of interest. However, in certain more global scenarios, e.g., in the situation of slow or inertial manifolds, it is possible to weaken (5) and replace it by a condition of the form (cf. $[33,34])$

$$
\sup _{t \in \mathbb{I}_{\tau}^{ \pm}(\infty)} \alpha_{ \pm}^{\tau-t}\|F(t, 0)\|<\infty
$$


Of central importance in this section are the following discrete Lyapunov-Perron operators $\mathscr{T}_{T}^{ \pm}: X_{\tau, \gamma}^{ \pm}(T) \times X \rightarrow X_{\tau, \gamma}^{ \pm}(T)$, which, for given $\xi \in X$, read as

$$
\begin{aligned}
& \mathscr{T}_{T}^{+}(\phi, \xi)=\Phi(\cdot, \tau) P_{+}(\tau) \xi+\sum_{r=\tau}^{\tau+T} G(\cdot, r+1) F(r, \phi(r)), \\
& \mathscr{T}_{T}^{-}(\phi, \xi)=\bar{\Phi}(\cdot, \tau) P_{-}(\tau) \xi+\sum_{r=\tau-T}^{\tau-1} G(\cdot, r+1) F(r, \phi(r)),
\end{aligned}
$$

respectively. Note that $T=\infty$ is explicitly allowed. Since the dependence of $\mathscr{T}_{T}^{ \pm}$ on $\tau$ is of minor importance in this paper, we have suppressed it. The corresponding respective fixed point problems

$$
\begin{array}{lll}
\phi=\Phi(\cdot, \tau) P_{+}(\tau) \xi+\sum_{r=\tau}^{\tau+T} G(\cdot, r+1) F(r, \phi(r)) & \text { in } X_{\tau, \gamma}^{+}(T), & \left(L P_{T}^{+}\right) \\
\phi=\bar{\Phi}(\cdot, \tau) P_{-}(\tau) \xi+\sum_{r=\tau-T}^{\tau-1} G(\cdot, r+1) F(r, \phi(r)) & \text { in } X_{\tau, \gamma}^{-}(T) & \left(L P_{T}^{-}\right)
\end{array}
$$

are denoted as discrete Lyapunov-Perron equations. They are related to the dynamical behavior of (2) as follows:

Proposition 1 Let $\tau \in \mathbb{I}, \xi \in X, \gamma \in\left(\alpha_{+}, \alpha_{-}\right)$and suppose Hypotheses 1-2 hold. If $\phi \in X_{\tau, \gamma}^{ \pm}(\infty)$ is a sequence satisfying

$$
L\left(\sup _{t \in \mathbb{I}_{\tau}^{ \pm}(\infty)}\|\phi(t)\|\right)<\infty
$$

then the following assertions are equivalent:

(a) $\phi$ solves the nonautonomous difference equation (2) with $P_{ \pm}(\tau) \phi(\tau)=\xi$,

(b) $\phi$ is a fixed point of the Lyapunov-Perron equations $\left(L P_{\infty}^{ \pm}\right)$.

Proof We restrict to the case $\phi \in X_{\tau, \gamma}^{+}(\infty)$, since the dual situation $\phi \in X_{\tau, \gamma}^{-}(\infty)$ can be treated similarly, and define $R:=\sup _{\tau \leq t}\|\phi(t)\|$. Let us consider sequences $\phi_{ \pm}(t):=P_{ \pm}(t) \phi(t)$ for $t \in \mathbb{I}_{\tau}^{+}(\infty)$.

$(a) \Rightarrow(b)$ If $\phi$ solves the difference equation (2), then $\phi_{+}$is a solution of the initial value problem

$$
x_{t+1}=A(t) P_{+}(t) x_{t}+P_{+}(t+1) F(t, \phi(t)), \quad x(\tau)=\xi
$$

and the discrete variation of constants formula yields $\phi_{+}(t)=P_{+}(t) \mathscr{T}_{T}^{+}(t, \phi, \xi)$ for all $t \geq \tau$. Moreover, by Hypotheses 1-2 and the triangle inequality, we have

$$
\left\|P_{-}(t+1)[F(t, \phi(t))]\right\| \gamma^{\tau-t} \stackrel{(5)}{\leq} K_{-} L(R)\|\phi(t)\| \gamma^{\tau-t} \leq 2 K_{-} L(R)\|\phi\|_{\tau, \gamma}^{+}
$$

for all $\tau \leq t$, and hence the inhomogeneous part of equation

$$
x_{t+1}=A(t) P_{-}(t) x_{t}+P_{-}(t+1) F(t, \phi(t))
$$


is exponentially bounded. By [33, Lemma 3.1(a)] this equation admits a unique solution $\phi_{-} \in X_{\tau, \gamma}^{+}(\infty)$, which additionally has the form $\phi_{-}(t)=P_{-}(t) \mathscr{T}_{T}^{-}(t, \phi, \xi)$ for all $\tau \leq t$. Then $\phi=\phi_{-}+\phi_{+}$solves the fixed point problem $\left(L P_{T}^{+}\right)$.

$(b) \Rightarrow(a)$ Conversely, let $\phi \in X_{\tau, \gamma}^{+}(\infty)$ be a solution of the Lyapunov-Perron equation $\left(L P_{T}^{+}\right)$. Then the discrete variation of constants formula implies that $\phi_{+}$ is the unique forward solution of the initial value problem (6). Furthermore, also [33, Lemma 3.1(a)] guarantees that $\phi_{-}$is an exponentially bounded solution of the linear inhomogeneous system (7).

Under stronger global conditions, we can establish the existence of unique solutions for the Lyapunov-Perron equations:

Proposition 2 Let $\tau \in \mathbb{I}, \xi \in X, T \in(0, \infty]_{\mathbb{Z}}$, suppose Hypotheses 1-2 hold with

$$
\ell:=\max \left\{K_{-}, K_{+}\right\} L(\infty)<\frac{\alpha_{-}-\alpha_{+}}{4}
$$

and choose $\sigma \in\left(\ell, \frac{1}{2}\left(\alpha_{-}-\alpha_{+}\right)\right]$. Then, for $\gamma \in\left[\alpha_{+}+\sigma, \alpha_{-}-\sigma\right]$, the LyapunovPerron equations $\left(L P_{T}^{ \pm}\right)$have a unique solution $\phi_{T}^{ \pm}(\xi) \in X_{\tau, \gamma}^{ \pm}(T)$ which satisfy

$$
\left\|\phi_{T}^{ \pm}(\xi)\right\|_{\tau, \gamma}^{ \pm} \leq \frac{K_{ \pm} \sigma}{\sigma-\ell}\left\|P_{ \pm}(\tau) \xi\right\|,
$$

and in addition, the sequences $\phi_{T}^{ \pm}(\xi)$ do not depend on $\gamma$.

In order not to overextend our notation, we also suppress the dependence of the sequences $\phi_{T}^{ \pm}(\xi) \in X_{\tau, \gamma}^{ \pm}(T)$ on the initial time $\tau \in \mathbb{I}$.

Proof Let $\tau \in \mathbb{I}$ and $\xi \in X$. We only sketch a proof and refer to [33] for the details. Thereto, consider the Lyapunov-Perron operator $\mathscr{T}_{T}^{ \pm}: X_{\tau, \gamma}^{ \pm}(T) \times X \rightarrow X_{\tau, \gamma}^{ \pm}(T)$. It can be verified as in [33, Lemma 3.2] that $\mathscr{T}_{T}^{ \pm}$is well-defined and satisfies the two Lipschitz estimates

$$
\operatorname{Lip}_{1} \mathscr{T}_{T}^{ \pm} \leq \frac{\ell}{\sigma}<1, \quad \operatorname{Lip}_{2} \mathscr{T}_{T}^{ \pm} \leq K_{ \pm} .
$$

From the first inequality in (9), we get that $\mathscr{T}_{T}^{ \pm}(\cdot, \xi)$ is a contraction on $X_{\tau, \gamma}^{ \pm}(T)$, uniformly in $\xi$, and Banach's fixed point theorem implies that there exists a unique fixed point $\phi_{T}^{ \pm}(\xi) \in X_{\tau, \gamma}^{ \pm}(T)$. Moreover, the second inequality in (9) yields the claimed bound on $\phi_{T}^{ \pm}(\xi)$.

We are in the position to introduce a nonautonomous counterpart to (global) invariant manifolds of autonomous difference equations (maps). A fiber bundle is a nonautonomous set $S$, where each fiber $S(t), t \in \mathbb{I}$, is graph of a function.

Theorem 1 (invariant fiber bundles) Assume Hypothesis 1-2 hold with (8) and choose $\sigma \in\left(\ell, \frac{1}{2}\left(\alpha_{-}-\alpha_{+}\right)\right]$. Then the following statements are true:

(a) If $\mathbb{I}$ is unbounded above, then the so-called pseudo-stable fiber bundle

$$
S^{+}:=\left\{(\tau, \xi) \in \mathbb{I} \times X: \varphi(\cdot ; \tau, \xi) \in X_{\tau, \gamma}^{+}(\infty) \text { for } \gamma \in\left[\alpha_{+}+\sigma, \alpha_{-}-\sigma\right]\right\}
$$


is a positively invariant fiber bundle of (2) possessing the representation

$$
S^{+}=\left\{\left(\tau, \xi+s^{+}(\tau, \xi)\right) \in \mathbb{I} \times X: \tau \in \mathbb{I}, \xi \in R\left(P_{+}(\tau)\right)\right\}
$$

with a uniquely determined mapping $s^{+}: \mathbb{I} \times X \rightarrow X$, given by

$$
s^{+}(\tau, \xi)=P_{-}(\tau) \phi_{\infty}^{+}(\tau, \xi) \quad \text { for all } \tau \in \mathbb{I}, \xi \in X .
$$

Furthermore, $s^{+}$satisfies $\operatorname{Lip}_{2} s^{+} \leq \frac{K_{-} K_{+} L}{\sigma-\ell}$ and one has $s^{+}(\tau, 0) \equiv 0$ on $\mathbb{I}$.

(b) If $\mathbb{I}$ is unbounded below, then the so-called pseudo-unstable fiber bundle

$$
S^{-}:=\left\{(\tau, \xi) \in \mathbb{I} \times X: \begin{array}{l}
\text { there exists a solution } \phi: \mathbb{I} \rightarrow X \text { of }(2) \text { with } \\
\phi(\tau)=\xi \text { and } \phi \in X_{\tau, \gamma}^{-}(\infty) \text { for } \gamma \in\left[\alpha_{+}+\sigma, \alpha_{-}-\sigma\right]
\end{array}\right\}
$$

is an invariant fiber bundle of (2) possessing the representation

$$
S^{-}=\left\{\left(\tau, \eta+s^{-}(\tau, \eta)\right) \in \mathbb{I} \times X: \tau \in \mathbb{I}, \eta \in R\left(P_{-}(\tau)\right)\right\}
$$

with a uniquely determined mapping $s^{-}: \mathbb{I} \times X \rightarrow X$, given by

$$
s^{-}(\tau, \eta)=P_{+}(\tau) \phi_{\infty}^{-}(\tau, \eta) \quad \text { for all } \tau \in \mathbb{I}, \eta \in X
$$

Furthermore, $s^{-}$satisfies $\operatorname{Lip}_{2} s^{-} \leq \frac{K_{-} K_{+} L}{\sigma-\ell}$ and one has $s^{-}(\tau, 0) \equiv 0$ on $\mathbb{I}$.

Proof See [33, Theorem 3.5].

Remark 3 It can be shown that time-periodic difference equations (2) admit fiber bundles $S^{ \pm}$with periodic fibers $S^{ \pm}(t)$. In particular, for autonomous equations these fibers are constant and we obtain the usual invariant manifolds.

Remark 4 Under very similar assumptions supplementing Hypothesis 2 (cf. [33, 34]) the above theorem also holds for implicit difference equations of the form

$$
x_{t+1}=A(t) x_{t}+F\left(t, x_{t}, x_{t+1}\right),
$$

which, for instance, have been obtained from fully-implicit discretizations of differential equations. Here, $S^{+}$consists of solutions, which exist and are exponentially bounded in forward time. With obvious modifications, our later tools also work and are applicable for such problems.

The nonautonomous sets $S^{+}$and $S^{-}$generalize the classical invariant manifolds corresponding to pseudo-hyperbolic equilibria; to be more specific and to provide a dynamic insight, $S^{+}$is called

- center-stable fiber bundle in case $\alpha_{-}>1$; it contains solutions bounded in forward time,

- stable fiber bundle in the hyperbolic situation $\alpha_{+}<1<\alpha_{-}$; it contains exponentially decaying forward solutions, and

- strongly stable fiber bundle in case $\alpha_{-}<1$.

Under the assumption of $\mathbb{I}$ being unbounded below, $S^{-}$is called

- center-unstable fiber bundle in case $\alpha_{+}<1$; it contains solutions which exist and are bounded in backward time, 
- unstable fiber bundle in the hyperbolic situation $\alpha_{+}<1<\alpha_{-}$; it contains solutions existing and exponentially decaying in backward time; and

- strongly unstable fiber bundle in case $1<\alpha_{+}$.

Detached from the idea of describing dynamics near isolated solutions, the sets $S^{ \pm}$yield the global set of all solutions with a specific boundedness behavior in forward or backward time. Hence, in case $\alpha_{-}<1$ one can interpret $S^{-}$as discrete inertial manifold (cf. [34]) and (8) as corresponding spectral gap condition.

\section{Computation of invariant fiber bundles}

The relations (10) and (11) are central for our approach to approximate the fiber bundles $S^{ \pm}$. Indeed, in order to compute the functions $s^{ \pm}$defining $S^{ \pm}$, we solve the Lyapunov-Perron equations $\left(L P_{T}^{ \pm}\right)$for $T<\infty$. The corresponding error estimate for the distance between the fixed points $\phi_{T}^{ \pm}(\tau, \xi)$ and $\phi_{\infty}^{ \pm}(\tau, \xi)$ is given in

Proposition 3 Let $\tau \in \mathbb{I}, \xi \in X, T \in \mathbb{N}$, suppose that Hypotheses 1-2 hold with (8), choose $\sigma \in\left(\ell, \frac{1}{2}\left(\alpha_{-}-\alpha_{+}\right)\right)$and set $L:=L(\infty)$. Then the function $s^{ \pm}: \mathbb{I} \times X \rightarrow X$ defining the fiber bundle $S^{ \pm}$satisfies

$$
\left\|s^{ \pm}(\tau, \xi)-P_{\mp}(\tau) \phi_{T}^{ \pm}(\tau, \xi)\right\| \leq \frac{\sigma K_{\mp}^{2} K_{ \pm} L}{(\sigma-\ell)^{2}}\left\|P_{ \pm}(\tau) \xi\right\|\left(\frac{\alpha_{+}+\sigma}{\alpha_{-}-\sigma}\right)^{T} .
$$

Remark 5 (spectral ratio condition) Keeping in mind that $\ell$ is supposed to be small, one can choose $\sigma$ close to 0 and the decay rate $\frac{\alpha_{+}+\sigma}{\alpha_{-}-\sigma}$ in (12) essentially depends on the ratio $\frac{\alpha_{+}}{\alpha_{-}}$. Thus, we obtain a good approximation for small values of $T>0$ in (12), provided $\frac{\alpha_{+}}{\alpha_{-}} \ll 1$. In the autonomous situation, this means that consecutive spectral points have moduli with small quotients.

Proof Due to analogy, we only prove the assertion for $s^{-}$and $\phi_{T}^{-}$. Choose a finite positive integer $T, \gamma \in\left(\alpha_{+}+\sigma, \alpha_{-}-\sigma\right]$, and thanks to $\sigma<\frac{1}{2}\left(\alpha_{-}-\alpha_{+}\right)$, we can select a $\delta \in\left[\alpha_{+}+\sigma, \gamma\right)$. Let $\tau \in \mathbb{I}, \xi \in X$ be fixed and $\phi_{T}^{-} \in X_{\tau, \gamma}^{-}(T), \phi_{\infty}^{-} \in X_{\tau, \gamma}^{-}(\infty)$ be the unique solutions of the Lyapunov-Perron equations $\left(L P_{T}^{-}\right)$and $\left(L P_{\infty}^{-}\right)$, respectively. Here we have suppressed the dependence on $\xi$. Then, on the finite interval $[\tau-T, \tau]_{\mathbb{Z}}$, one evidently has $\phi_{T}^{-},\left.\phi_{\infty}^{-}\right|_{\mathbb{I}_{\tau}^{-}(T)} \in X_{\tau, \delta}^{-}(T)$, and we obtain from Proposition 2 that

$$
\begin{aligned}
& \left\|P_{+}(t)\left[\phi_{\infty}^{-}(t)-\phi_{T}^{-}(t)\right]\right\| \delta^{\tau-t} \leq\left\|\sum_{r=-\infty}^{\tau-1-T} \Phi(t, r+1) P_{+}(r+1) F\left(r, \phi_{\infty}^{-}(r)\right)\right\| \delta^{\tau-t} \\
& +\left\|\sum_{r=\tau-T}^{t-1} \Phi(t, r+1) P_{+}(r+1)\left[F\left(r, \phi_{\infty}^{-}(r)\right)-F\left(r, \phi_{T}^{-}(r)\right)\right]\right\| \delta^{\tau-t} \\
\leq & \frac{K_{+} L}{\alpha_{+}} \sum_{r=-\infty}^{\tau-1-T} \alpha_{+}^{t-r}\left\|\phi_{\infty}^{-}(r)\right\| \delta^{\tau-t}+\frac{K_{+} L}{\alpha_{+}} \sum_{r=\tau-T}^{t-1} \alpha_{+}^{t-r}\left\|\phi_{\infty}^{-}(r)-\phi_{T}^{-}(r)\right\| \delta^{\tau-t} \\
\leq & \frac{K_{+} L}{\gamma-\alpha_{+}}\left(\frac{\delta}{\gamma}\right)^{T}\left\|\phi_{\infty}^{-}\right\|_{\tau, \gamma}^{-}+\frac{K_{+} L}{\delta-\alpha_{+}}\left\|\phi_{\infty}^{-}-\phi_{T}^{-}\right\|_{\tau, \delta}^{-} \quad \text { for all } t \in[\tau-T, \tau]_{\mathbb{Z}},
\end{aligned}
$$


and similarly,

$$
\left\|P_{-}(t)\left[\phi_{\infty}^{-}(t)-\phi_{T}^{-}(t)\right]\right\| \delta^{\tau-t} \leq \frac{K_{-} L}{\alpha_{-}-\delta}\left\|\phi_{\infty}^{-}-\phi_{T}^{-}\right\|_{\tau, \delta}^{-} \quad \text { for all } t \in[\tau-T, \tau]_{\mathbb{Z}} .
$$

By definition of the $\|\cdot\|_{\tau, \delta}^{-}$-norm and due to $\gamma, \delta \in\left[\alpha_{+}+\sigma, \alpha_{-}-\sigma\right]$, we arrive at

$$
\left\|\phi_{T}^{-}-\phi_{\infty}^{-}\right\|_{\tau, \delta}^{-} \leq \frac{K_{+} L}{\sigma}\left(\frac{\delta}{\gamma}\right)^{T}\left\|\phi_{\infty}^{-}\right\|_{\tau, \delta}^{-}+\frac{\ell}{\sigma}\left\|\phi_{T}^{-}-\phi_{\infty}^{-}\right\|_{\tau, \delta}^{-}
$$

and consequently (note the inequality $\ell<\sigma$ ),

$$
\left\|P_{+}(t)\left[\phi_{\infty}^{-}(t)-\phi_{T}^{-}(t)\right]\right\| \delta^{\tau-t} \leq \frac{K_{+}^{2} L}{\sigma-\ell}\left(\frac{\delta}{\gamma}\right)^{T}\left\|\phi_{\infty}^{-}\right\|_{\tau, \gamma}^{-} \quad \text { for all } t \in[\tau-T, \tau]_{\mathbb{Z}} .
$$

Therefore, the claim follows from Proposition 2(b), if we use (11) and set $t=\tau$, $\delta=\alpha_{+}+\sigma, \gamma=\alpha_{-}-\sigma$ in the above estimate.

\subsection{Computation of single points}

Having these error estimates at hand, we are in a position to solve the truncated fixed point equations $\left(L P_{T}^{ \pm}\right)$instead of $\left(L P_{\infty}^{ \pm}\right)$for some fixed $T>0$. So we reduce the infinite-dimensional problem $\left(L P_{\infty}^{ \pm}\right)$to a nonlinear algebraic equation.

To approximate the pseudo-unstable fiber bundle $S^{-}$, we fix an initial point $\xi \in X$ and proceed with another simplification. Multiplying the Lyapunov-Perron equation $\left(L P_{T}^{-}\right)$with projections $P_{+}(t)$ and $P_{-}(t)$ implies

$$
\begin{aligned}
& \psi^{+}(t)=\sum_{r=\tau-T}^{t-1} \Phi(t, r+1) P_{+}(r+1) F\left(r, \psi^{+}(r)+\psi^{-}(r)\right), \\
& \psi^{-}(t)=\bar{\Phi}(t, \tau) P_{-}(\tau) \xi-\sum_{r=t}^{\tau-1} \bar{\Phi}(t, r+1) P_{-}(r+1) F\left(r, \psi^{+}(r)+\psi^{-}(r)\right),
\end{aligned}
$$

respectively, where we have abbreviated $\psi^{ \pm}(t)=P_{ \pm}(t) \phi_{T}^{-}(t, \xi)$. In particular, we have the relation $\psi^{-}(\tau)=P_{-}(\tau) \xi$. The discrete variation of constants formula guarantees that $\psi^{-}$is a backward solution of the difference equation

$$
x_{t+1}=A(t) P_{-}(t) x_{t}+P_{-}(t+1) F\left(t, x_{t}+\psi^{+}(t)\right),
$$

and we simplified $\left(L P_{T}^{-}\right)$to the following algebraic system of nonlinear equations

$$
\begin{gathered}
\psi^{+}(t)-\sum_{r=\tau-T}^{t-1} \Phi(t, r+1) P_{+}(r+1) F\left(r, \psi^{+}(r)+\psi^{-}(r)\right)=0 \\
\quad \text { for all } t \in[\tau-T, \tau]_{\mathbb{Z}}, \\
\psi^{-}(t+1)-A(t) \psi^{-}(t)-P_{-}(t+1) F\left(t, \psi^{+}(t)+\psi^{-}(t)\right)=0 \\
\quad \text { for all } t \in[\tau-T, \tau-1]_{\mathbb{Z}}, \\
\psi^{-}(\tau)=P_{-}(\tau) \xi .
\end{gathered}
$$


The first equation in (13) degenerates into $\psi^{+}(\tau-T)=0$ for $t=\tau-T$, which causes no confusion, since (13) is used to obtain $P_{ \pm}(t) \phi_{\infty}^{-}(t, \xi)$ only for $t=\tau$.

Next we further transform (13) into a form more appropriate for implementation on a computer. Thereto, we suppose $d:=\operatorname{dim} X<\infty$ and conclude that (13) becomes a $(1+T) d$-dimensional problem. We fix $\tau \in \mathbb{I}$, choose respective bases $e_{1}^{+}, \ldots, e_{d_{+}}^{+}$of $R\left(P_{+}(\tau)\right)$ and $e_{1}^{-}, \ldots, e_{d_{-}}^{-}$of $R\left(P_{-}(\tau)\right)$ (note that $d_{+}$and $d_{-}$are independent of $\tau$ ) and introduce scalar variables $x_{1}, \ldots, x_{(1+T) d}$ according to

$$
\begin{aligned}
y_{n} & =\sum_{j=1}^{d_{+}} x_{j+d_{+}(n-1)} e_{j}^{+}=\psi^{+}(\tau-T+n-1), \\
y_{n+T+1} & =\sum_{j=1}^{d_{-}} x_{j+d_{-}(n-1)+d_{+}(T+1)} e_{j}^{-}=\psi^{-}(\tau-T+n-1)
\end{aligned}
$$

for all $n=1, \ldots, T+1$. With this notation we can write (13) as

$$
\begin{aligned}
& y_{t+1+T}-\sum_{r=-T}^{t-1} \Phi(t+\tau, r+\tau+1) P_{+}(r+\tau+1) F\left(r+\tau, y_{r+T+1}+y_{r+2 T+2}\right)=0 \\
& \text { for all } t \in[-T, 0]_{\mathbb{Z}}, \\
& y_{t+2 T+3}-A(t+\tau) y_{t+2 T+2}-P_{-}(t+\tau+1) F\left(t+\tau, y_{t+T+1}+y_{t+2 T+2}\right)=0 \\
& \text { for all } t \in[-T,-1]_{\mathbb{Z}}, \\
& y_{2 T+2}=P_{-}(\tau) \xi,
\end{aligned}
$$

or in an even more compact notation abstractly as

$$
G^{-}(x, \xi)=0 \quad \text { with } x \in \mathbb{K}^{(1+T) d} \text { and } \xi \in \mathbb{K}^{d_{-}} .
$$

Each computation of $G^{-}(\cdot, \xi)$ involves $\frac{T}{2 d}\left(d_{+} T+d+d_{-}\right)$evaluations of $F$.

For the corresponding dual approximation method of the pseudo-stable fiber bundle $S^{+}$, we set $\psi^{ \pm}(t)=P_{ \pm}(t) \phi_{T}^{+}(t, \xi)$, and $\left(L P_{T}^{+}\right)$reduces to

$$
\begin{gathered}
\psi^{+}(\tau)=P_{+}(\tau) \xi \\
\psi^{+}(t+1)-A(t) \psi^{+}(t)-P_{+}(t+1) F\left(t, \psi^{+}(t)+\psi^{-}(t)\right)=0 \\
\quad \text { for all } t \in[\tau, \tau+T-1]_{\mathbb{Z}}, \\
\psi^{-}(t)+\sum_{r=t}^{\tau+T} \Phi(t, r+1) P_{-}(r+1) F\left(r, \psi^{+}(r)+\psi^{-}(r)\right)=0 \\
\text { for all } t \in[\tau, \tau+T]_{\mathbb{Z}} .
\end{gathered}
$$

With the above notation, we now introduce scalars $x_{1}, \ldots, x_{(1+T) d}$ according to

$$
\begin{aligned}
y_{n} & =\sum_{j=1}^{d_{+}} x_{j+d_{+}(n-1)} e_{j}^{+}=\psi^{+}(\tau+n-1), \\
y_{n+T+1} & =\sum_{j=1}^{d_{-}} x_{j+d_{-}(n-1)+d_{+}(T+1)} e_{j}^{-}=\psi^{-}(\tau+n-1)
\end{aligned}
$$


for all $n=1, \ldots, T+1$, and write (14) as

$$
\begin{gathered}
y_{1}=P_{+}(\tau) \xi \\
y_{t+1}-A(t-1+\tau) y_{t}-P_{+}(t+\tau) F\left(t-1+\tau, y_{t}+y_{t+1+T}\right)=0 \\
\quad \text { for all } t \in[1, T]_{\mathbb{Z}}, \\
y_{t+T+2}+\sum_{r=t}^{T} \Phi(t+\tau, r+\tau+1) P_{-}(r+\tau+1) F\left(r+\tau, y_{r+1}+y_{r+T+2}\right)=0 \\
\quad \text { for all } t \in[0, T]_{\mathbb{Z}},
\end{gathered}
$$

which we formally also write as

$$
G^{+}(x, \xi)=0 \quad \text { with } x \in \mathbb{K}^{(1+T) d} \text { and } \xi \in \mathbb{K}^{d_{+}} .
$$

Now, each computation of $G^{+}(\cdot, \xi)$ involves $\frac{1}{2 d}\left[2 d_{+} T+d_{-}(T+1)(T+2)\right]$ evaluations of the nonlinearity $F$.

We denote the unique solution of the nonlinear algebraic equations (13) and (14) (cf. Proposition 2) by $\Psi_{\infty}^{ \pm}(\xi)$, resp., and get

Algorithm 1 (approximation of $s^{ \pm}(\tau, \xi)$ ) Choose a desired accuracy $\varepsilon>0$ and values $\tau \in \mathbb{I}, \xi \in R\left(P_{ \pm}(\tau)\right), \sigma \in\left(\ell, \frac{1}{2}\left(\alpha_{-}-\alpha_{+}\right)\right)$.

(1) Set $n:=0, \Psi_{0}^{ \pm}:=0$ and an integer $T>0$ so large that

$$
\frac{\sigma K_{\mp}^{2} K_{ \pm} L}{(\sigma-\ell)^{2}}\left\|P_{ \pm}(\tau) \xi\right\|\left(\frac{\alpha_{+}+\sigma}{\alpha_{-}-\sigma}\right)^{T}<\frac{\varepsilon}{2}
$$

(2) apply an iterative numerical method to (13) and (14), respectively, in order to obtain an approximation $\Psi_{n+1}^{ \pm}$from $\Psi_{n}^{ \pm}$

(3) if $\left\|\Psi_{\infty}^{ \pm}-\Psi_{n}^{ \pm}\right\| \geq \frac{\varepsilon}{2}$, then increase $n$ by 1 and go to (2)

(4) $\operatorname{set} \tilde{s}^{ \pm}(\tau, \xi):=\Psi_{n}^{+}(\tau)$.

By construction of this algorithm, the distance between the approximate invariant fiber bundle $\tilde{s}^{ \pm}(\tau, \xi)$ and $s^{ \pm}(\tau, \xi)$ satisfies

$$
\left\|s^{ \pm}(\tau, \xi)-\tilde{s}^{ \pm}(\tau, \xi)\right\|<\varepsilon .
$$

From a numerical perspective, the crucial point in Algorithm 1 is of course an appropriate choice of the iterative method in step (2) to solve the parameterdependent nonlinear equations (13) and (14), respectively, i.e.,

$$
G^{ \pm}(x, \xi)=0 .
$$

The function $G^{ \pm}$inherits its smoothness properties from the nonlinearity $F$, and due to Hypothesis 1 , in general $G^{ \pm}$is only globally Lipschitz continuous in the first variable. Therefore, a universally applicable approach to solve (16) is to transform this equation into a fixed-point problem and use fixed-point iteration. Clearly, this method is only linearly convergent and one prefers methods with better convergence properties. Newton-like methods are formally applicable to (16), if $G^{ \pm}$ is at least differentiable and a more detailed description of the algorithms used here can be found in the beginning of the following Section 4 . 
Remark 6 (nonsmooth methods) By Rademacher's theorem, the Lipschitz functions $G^{ \pm}$are differentiable almost everywhere and the last two decades saw a strong interest in generalized Newton methods for nonsmooth equations (see [36] for a survey). They are typically of the form

$$
x_{n+1}=x_{n}-V_{n}^{-1} G^{ \pm}\left(x_{n}, \xi\right), \quad V_{n} \in \partial_{1} G^{ \pm}\left(x_{n}, \xi\right),
$$

where the set $\partial_{1} G^{ \pm}\left(x_{n}, \xi\right)$ is the generalized Jacobian of $G^{ \pm}(\cdot, \xi)$ at $x_{n}$, defined by [7], and $V_{n}$ is arbitrarily taken from $\partial_{1} G^{ \pm}\left(x_{n}, \xi\right)$. Unfortunately, for only Lipschitzian mappings $G^{ \pm}$, the iteration (17) needs not to converge (see [27] for a counter-example). Locally superlinear convergence results have been obtained by [40] under the assumption that $G^{ \pm}$is semismooth (see [29]). This class contains functions with piecewise linear, differentiable or convex components, and is closed under addition and multiplication.

Remark 7 (structure of the Jacobian) In Figure 1 we sketched the structure of the Jacobian $D_{1} G^{+}(x, \xi)$, where zero elements are not included. While it is not sparse, it indicates that such problems might be appropriate for decomposition methods.
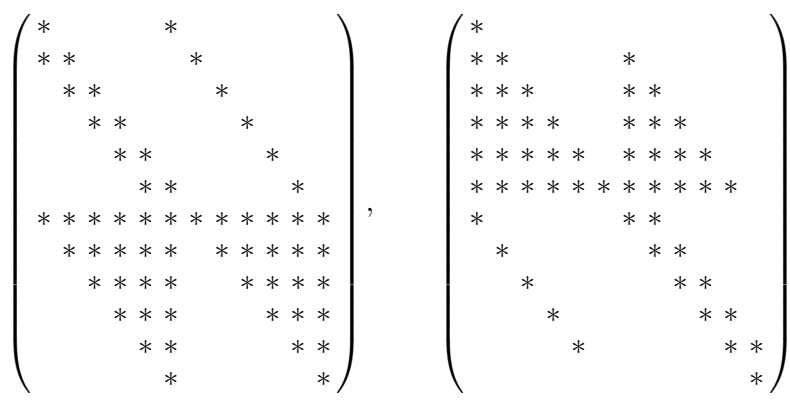

Fig. 1 Structure of the Jacobians $D_{1} G^{+}(x, \xi)$ (left) and $D_{1} G^{-}(x, \xi)$ (right) for $T=5$

\subsection{Computation of fibers}

The Algorithm 1 is designed to approximate single points on fixed fibers of invariant fiber bundles. To obtain individual fibers as a whole, one could compute various points and interpolate afterwards.

An alternative approach is the use of continuation methods: For given initial time $\tau \in \mathbb{I}$, Algorithm 1 yields an approximation of $s^{ \pm}\left(\tau, \xi_{0}\right)$ for a fixed value of $\xi_{0}$. In order to compute $s^{ \pm}(\tau, \xi)$ for different values of $\xi$, it is contiguous to use a continuation (or path following) algorithm applied to (16) (see [1] for an overview). For our further description of this procedure, we suppose that $G^{ \pm}$is sufficiently smooth and the parameter $\xi$ is scalar, i.e., we deal with 1-dimensional fibers. Upon differentiating the identity (16) and due to our particular parameter dependence, a so-called Davidenko differential equation is obtained:

$$
D_{1} G^{ \pm}(x(\xi), \xi) \frac{d x(\xi)}{d \xi}= \begin{cases}e_{1}, & \text { if } s^{+} \text {is considered } \\ e_{(T+1) d}, & \text { if } s^{-} \text {is considered }\end{cases}
$$


This is an implicit ordinary differential equation and our desired function $x$ is a solution satisfying the initial condition $x(\tau)=\xi_{0}$, where the initial point $\xi_{0}$ can be obtained using Algorithm 1. However, for various reasons it is not advantageous to tackle (18) directly using an ODE solver. One alternative is a classical continuation method as described in the following algorithm:

Algorithm 2 (classical path-following) Choose an accuracy $\varepsilon>0, T>0$, stepsize bounds $0<h_{\min }<h_{\max }$, an initial step-size $h>h_{\min }$, values $\tau \in \mathbb{I}, \xi_{0} \in \mathbb{R}$ and $\xi_{\max }>\xi_{0}$.

(1) Set $n:=0$ and compute a solution $x_{0}$ of $G^{ \pm}\left(x, \xi_{0}\right)=0$ using Algorithm 1

(2) set $\xi_{n+1}=\xi_{n}+h$

(3) solve $G^{ \pm}\left(x, \xi_{n+1}\right)=0$ with $x_{n}$ as initial iterate to obtain $x_{n+1}$

(4) if $\left\|G^{ \pm}\left(x_{n+1}, \xi\right)\right\|<\varepsilon$ then

increase $n$ by 1 ,

set $h=\min \left\{h_{\max }, 1.5 h\right\}$

else $h=0.5 h$

(5) if $h>h_{\min }$ and $\xi<\xi_{\max }$, then go to (2).

Appropriate components of the obtained vectors $x_{n}$ approximate $s^{ \pm}(\tau, \cdot)$ at discrete points $\xi_{n}$ and the whole fiber $S^{ \pm}(\tau)$ can be obtained using interpolation.

While the above Algorithm 2 includes a crude step-size control, we refer to [11, Chapter 5] for a more sophisticated approach. Such a continuation method can compute fibers $S^{ \pm}(\tau)$ representable as graphs over the linear spaces $R\left(P^{ \pm}(\tau)\right)$.

On the other hand, to approximate fibers given as embedded manifolds, we use pseudo-arclength continuation as follows. We define $u:=(x, \xi)$, and choosing a scalar arclength increment $h>0$, we work with the extended equation

$$
\tilde{G}_{h}^{ \pm}(u):=\left(\begin{array}{c}
G^{ \pm}(u) \\
N_{\tilde{u}}^{h}(u)
\end{array}\right)=\left(\begin{array}{l}
0 \\
0
\end{array}\right),
$$

where $N_{\tilde{u}}^{h}(u):=\dot{\tilde{u}}(u-\tilde{u})-h$.

The vector $\dot{\tilde{u}}$ represents the tangential approximation of length 1 of the solution curve in the current point $\tilde{u}$, and hence, the equation $N_{\tilde{u}}^{h}(u)=0$ means that the new point on the path lies on the tangent vector through the current point $\tilde{u}$.

The pseudo-arclength continuation algorithm can then be described as follows.

Algorithm 3 (pseudo-arclength path-following) Choose an accuracy $\varepsilon>0, T>$ 0 , step-size bounds $0<h_{\min }<h_{\max }$, an initial step-size $h>h_{\min }$, values $\tau \in \mathbb{I}$ and $s_{\text {max }}>0$.

(1) Set $n:=0, s:=0$ and $u_{0}:=0$

(2) compute the direction $\tilde{u}_{n}$ or choose $\tilde{u}_{0}$ in case $n=0$

(3) compute a solution $u_{n+1}$ of $\tilde{G}_{h}^{ \pm}\left(u_{n}\right)=0$ using an iterative numerical algorithm

(4) if $\left\|G^{ \pm}\left(u_{n+1}\right)\right\|<\varepsilon$ then increase $n$ by 1

set $h=\min \left\{h_{\max }, 1.5 h\right\}$

increase $s$ by $h$

else $h=0.5 h$

(5) if $h>h_{\min }$ and $s<s_{\max }$, then go to (2). 
Appropriate interpolation of components of the obtained vectors $u_{n}$ approximate the $\tau$-fiber of the invariant fiber bundle, i.e., this yields an approximation to the solution curve $\gamma: \mathbb{R} \rightarrow \mathbb{K}^{(1+T) d+1}$ satisfying

$$
G^{ \pm}(\gamma(s)) \equiv 0 \quad \text { for all } s \in\left[0, s_{\max }\right] .
$$

A dual algorithm leads to an approximation on the interval $\left[s_{\min }, 0\right]$.

\subsection{Approximation with one-step methods}

We finally mention how our discrete methods can be applied to compute invariant or integral manifolds of semi-linear ordinary differential equations

$$
\dot{x}=A(t) x+F(t, x) .
$$

As time steps, let $\left(t_{k}\right)_{k \in \mathbb{Z}}$ denote a strictly increasing sequence of real numbers with $\lim _{k \rightarrow \pm \infty} t_{k}= \pm \infty$. We apply a one-step method

$$
x_{k+1}=x_{k}+\left(t_{k+1}-t_{k}\right) \Psi\left(t_{k}, x_{k}, t_{k+1}-t_{k}\right)
$$

(e.g., of Runge-Kutta type) to (19). Then (20) can be written as semi-linear difference equation of the form (2). Under consistency and convergence assumptions on (20) this recursion can be shown to satisfy Hypotheses 1-2 for sufficiently small maximal step-sizes $t_{k+1}-t_{k}$.

Consequently, we can use the previous methodology in order to compute the invariant fiber bundles $\hat{S}^{ \pm}$of the one-step method (20) applied to our original continuous problem (19). Results to estimate the difference between the integral manifolds $S^{ \pm}$for (19) and the invariant fiber bundles $\hat{S}^{ \pm}$of (20) date back to the pioneering contributions [3] (stable and unstable manifolds), [4] (center manifolds) and also [14] (pseudo-stable and -unstable manifolds); corresponding results for nonautonomous equations and varying step-sizes can be found in [21].

\section{Examples and illustrations}

Our approach to the computation of nonautonomous invariant manifolds approximates solutions on pseudo-stable or -unstable fiber bundles. Hence, it is not surprising that these sequences and therefore the solution components for the nonlinear algebraic equation (16) admit exponentially decaying components. More precisely, this motivates a preconditioning strategy:

Remark 8 The solutions $x \in \mathbb{R}^{(T+1) d}$ of (16) appear to show the following behavior: There exist $d$ blocks of length $T+1$ such that the components of each block decay exponentially. Thus, in order to avoid numerical instabilities, it is reasonable to use the following preconditioning strategy. Instead of (16) one considers the nonlinear system

$$
\gamma^{i-1} G_{i+(T+1) n}^{ \pm}(x, \xi)=0 \quad \text { for all } i=1, \ldots, T+1, n=0, \ldots, d-1 .
$$

with an appropriate damping parameter $\gamma>0$. Note that here $G_{j}^{ \pm}$means the $j$ th component of $G^{ \pm}(x, \xi) \in \mathbb{K}^{(1+T) d}$. 
Before illustrating our results we briefly describe the algorithms and codes used for our numerical computations, i.e., the solvers for the nonlinear system (16). For implementation purposes, we relied on the MatLab numerical computing environment (release R2007a). Throughout, the Jacobians of the nonlinear system (16) have been approximated using forward differences with step-size $\varepsilon=10^{-6}$. The interested reader is strongly encouraged to contact the authors to obtain our corresponding codes.

IMMOptiBox A helpful MatLab toolbox for optimization and data fitting has been developed under Hans Bruun Nielsen at the Informatics and Mathematical Modelling department of the Technical University of Denmark. It is called IMMOptiBox and can be downloaded under the URL

$$
\text { http://www2.imm.dtu.dk/ hbn/immoptibox/ }
$$

We worked with Version 1.6 from November 2006. The following algorithms have been implemented:

- I_LM (based on smarquardt) This function uses a Levenberg-Marquardt damping of a secant version for the Gauss-Newton method with parameters params $=\left[\right.$ temp $\left., \varepsilon, 0.01 \varepsilon, 300,10^{-6}\right]$, where temp serves as dummy.

- I_LM_C: Apply I_LM to the preconditioned system (16) from Remark 8.

- I_PDL (based on nonlinsys) This function implements a Powell's dog-leg algorithm with params $=\left[\right.$ temp $\left., \varepsilon, 0.01 \varepsilon, 300,10^{-6}\right]$ and initial trust region radius temp $=0.1\left(1+\left\|x_{0}\right\|\right)$ with starting vector $x_{0}$.

- I_PDL_C: Apply I_PDL to the preconditioned system (16) of Remark 8.

Kelley The monograph [22] is an excellent source for modern Newton-like methods. Moreover, the algorithms discussed herein are available for download at

$$
\text { http://www.siam.org/books/fa01/ }
$$

For given $\varepsilon>0$ we have chosen absolute and relative error tolerances according to the parameters tol $=[0.1 \varepsilon, \varepsilon]$. We refer to [22] for a more detailed description of the subsequent algorithms including their parameters.

- K_NSold (based on nsold): A Newton-Armijo nonlinear solver, where the Jacobians are factored using Gaussian elimination in the following variants:

- K_NSold0: params $=[40,-1,0.5,1]$

- K_NSold1: params $=[40,1,0,1]($ Newton method $)$

- K_NSold2: params $=[40,-1,1,1]($ chord method $)$

- K_NSold3: params $=[40,1,1,1]$ (Shamanskii method with one step per Jacobian evaluation)

- K_NSoli (based on nsoli): Inexact Newton-Armijo iteration with EisenstatWalker forcing term and parabolic line search via three point interpolation. We implemented the following variants:

- K_NSoli1: params $=[40,40,0.9,1,20]$ (GMRES as iteration)

- K_NSoli2: params $=[40,40,0.9,2,20]$ (GMRES $(m)$ as iteration)

- K_NSoli3: params $=[40,40,0.9,4,20]$ (TFQMR as iteration)

- K_NSoli4: params $=[40,40,0.9,3,20]$ (BICGSTAB as iteration)

- K_BrSola (based on brsola): globally convergent Broyden's method solver using Armijo rule with one vector storage and params $=[40,40]$. 
MatLab itself offers the routine fsolve as part of its Optimization toolbox to solve systems of nonlinear equations.

- M_DL: Trust-region dog-leg method (NonlEqnAlgorithm='dogleg')

- M_GN: Gauss-Newton method (NonlEqnAlgorithm=' gn')

- M_GN1: LineSearchType=' quadcubic' (line search algorithm uses a safeguarded mixed quadratic and cubic polynomial interpolation and extrapolation method)

- M_GN2: LineSearchType=' cubicpoly' (safeguarded cubic polynomial method which generally requires fewer function evaluations but more gradient evaluations. Thus, if gradients are being supplied and can be calculated inexpensively, the cubic polynomial line search method is preferable)

- M_LM: Levenberg-Marquardt method (NonlEqnAlgorithm=' Im')

- M_LM1: LineSearchType= 'quadcubic'

- M_LM2: LineSearchType=' cubicpoly'

\subsection{A discrete test example}

We start with an example which is so simple that we analytically know its invariant manifolds and can explicitly determine parameters needed for the error estimates. For appropriate choice of the linear part we can interpret its pseudo-stable manifold as stable, strongly stable and strongly unstable manifold, respectively.

Let $a, b$ be real numbers with $0<|a|<|b|$ and consider the 2-dimensional autonomous difference equation ${ }^{1}$

$$
\left\{\begin{array}{l}
x_{t+1}=a x_{t}+x_{t}^{2} \\
y_{t+1}=b y_{t}+\left(a^{2}-b\right) x_{t}^{2}+2 a x_{t} y_{t}+y_{t}^{2}
\end{array} .\right.
$$

This problem fits into the framework of Section 2 with diagonal linear part

$$
A=\left(\begin{array}{ll}
a & 0 \\
0 & b
\end{array}\right),
$$

possessing an exponential dichotomy with $\alpha_{+}=|a|, \alpha_{-}=|b|, K_{ \pm}=1$ and

$$
P_{+}=\left(\begin{array}{ll}
1 & 0 \\
0 & 0
\end{array}\right), \quad P_{-}=\left(\begin{array}{ll}
0 & 0 \\
0 & 1
\end{array}\right) .
$$

Moreover, the nonlinearity for (21) is given by

$$
F(x, y)=\left(\begin{array}{c}
x^{2} \\
\left(a^{2}-b\right) x^{2}+2 a x y+y^{2}
\end{array}\right) .
$$

1 An analogous analysis can be done for the system

$$
\left\{\begin{array}{l}
x_{t+1}=a x_{t}+x_{t}^{2}+2 b x_{t} y_{t}+\left(b^{2}-a\right) y_{t}^{2} \\
y_{t+1}=b y_{t}+y_{t}^{2}
\end{array}\right.
$$

with pseudo-stable and -unstable manifold given by $s^{+}(x) \equiv 0, s^{-}(y)=y^{2}$, respectively. 
If we equip $\mathbb{R}^{2}$ with the norm $\|(x, y)\|:=\max \{|x|,|y|\}$, then the induced matrix norm of the Jacobian is given by

$$
\|D F(x, y)\|=2 \max \left\{|x|+\left|\left(a^{2}-b\right) x+2 a y\right|,|a x+y|\right\},
$$

and using the mean value theorem, we arrive at the Lipschitz estimate

$$
\|F(x, y)-F(\bar{x}, \bar{y})\| \leq 2 \rho M(a, b)\left\|\left(\begin{array}{l}
x-\bar{x} \\
y-\bar{y}
\end{array}\right)\right\| \quad \text { for all } x, \bar{x}, y, \bar{y} \in[-\rho, \rho],
$$

with the real constant

$$
M(a, b):=\left\{\begin{array}{l}
1+a^{2}+a-b, \quad \text { if } a^{2} \geq b, a \geq 0 \\
\max \left\{1+a^{2}-a-b,|1-a|\right\}, \quad \text { if } a^{2} \geq b, a<0 \\
1-a^{2}+a+b, \quad \text { if } a^{2}<b, a \geq 0 \\
\max \left\{1+b-a^{2}-a,|1+a|\right\}, \quad \text { if } a^{2}<b, a<0
\end{array} .\right.
$$

In order to fulfill our assumptions from Theorem 1 , we set $\rho=\frac{\alpha_{-}-\alpha_{+}}{9 M(a, b)}$, obtain

$$
\ell=L=2 \rho M(a, b)=\frac{\alpha_{-}-\alpha_{+}}{4.5}<\frac{\alpha_{-}-\alpha_{+}}{4}
$$

and choose $\sigma=\frac{\alpha_{-}-\alpha_{+}}{5}$. It is not difficult to see that the pseudo-stable and pseudounstable manifold of (21), respectively, is given by

$$
s^{+}(x)=x^{2}, \quad s^{-}(y) \equiv 0 .
$$

In order to solve the nonlinear systems $G^{+}(x, \xi)=0$ for $s^{+}$, we individually applied and tested various algorithms to the hyperbolic, strongly stable and strongly unstable case. From a numerical perspective, these cases get increasingly difficult.

\subsubsection{The hyperbolic case}

The system parameters $a, b$, the error tolerance $\varepsilon$ and the constants $\ell, \sigma, \rho, T$ described above, are given by the following table. In particular, $T>0$ is chosen according to Proposition 3 such that the error is less than $\varepsilon$ on the interval $[-1,1]$.

\begin{tabular}{ccc|cccc}
$a$ & $b$ & $\varepsilon$ & $\ell$ & $\sigma$ & $\rho$ & $T$ \\
\hline$-1 / 2$ & 2 & $10^{-5}$ & $1 / 3$ & 0.3 & 0.051282 & 22
\end{tabular}

We introduce various parameters describing the performance of the algorithms:

- $\xi_{\min }, \xi_{\max }$ : Starting at initial points $\xi_{-}<0<\xi_{+}$, we used the classical continuation Algorithm 2 to obtain the maximal convergence interval $\left[\xi_{\min }, \xi_{\max }\right]$ for the different methods, where $\varepsilon$ is given as above, $h=0.1, h_{\min }=0.1 / 2^{5}$. Here, $\xi_{\max }$ is the maximal value such that $\left\|G^{+}\left(x, \xi_{\max }\right)\right\|<\varepsilon$ and $h>h_{\min }$, and $\xi_{\min }$ is defined correspondingly.

- res: Arithmetic mean of the $\ell^{2}$-residual

$$
\text { res }:=\frac{1}{n} \sum_{i=1}^{n}\left\|G^{+}\left(x, \xi_{i}\right)\right\|_{2}
$$

over $n$ uniformly distributed points $\xi_{i}$ in an interval $I$ (note that for the evaluation of the $\ell^{2}$-residual, we used Algorithm 1, i.e., made no step-size adaptions). 
- err: Arithmetic mean of the absolute error over $n$ uniformly distributed points in an interval $I$.

- eval: Arithmetic mean of the desired number of evaluations of $G^{+}$over $n$ uniformly distributed points in an interval $I$.

For $\xi_{-}=-0.1, \xi_{+}=0.1, I=[-0.5,0.5]$ and $n=101$, we obtain

\begin{tabular}{l|rrrrr} 
method & res & err & eval & $\xi_{\min }$ & $\xi_{\max }$ \\
\hline I_LM & $5.11 \mathrm{e}-6$ & $1.65 \mathrm{e}-6$ & 57 & $-9.89 \mathrm{e}-1$ & $1.48 \mathrm{e}+0$ \\
I_PDL & $4.98 \mathrm{e}-6$ & $1.94 \mathrm{e}-6$ & 56 & $-9.94 \mathrm{e}-1$ & $1.49 \mathrm{e}+0$ \\
K_BrSola & $1.74 \mathrm{e}-6$ & $1.50 \mathrm{e}-8$ & 16 & $-9.10 \mathrm{e}-1$ & $1.40 \mathrm{e}+0$ \\
K_NSoli1 & $9.80 \mathrm{e}-5$ & $8.01 \mathrm{e}-9$ & 23 & $-9.92 \mathrm{e}-1$ & $1.49 \mathrm{e}+0$ \\
K_NSoli2 & $9.80 \mathrm{e}-5$ & $8.01 \mathrm{e}-9$ & 23 & $-9.92 \mathrm{e}-1$ & $1.49 \mathrm{e}+0$ \\
K_NSoli3 & $5.68 \mathrm{e}-7$ & $1.14 \mathrm{e}-10$ & 24 & $-9.92 \mathrm{e}-1$ & $1.49 \mathrm{e}+0$ \\
K_NSoli4 & $4.96 \mathrm{e}-7$ & $5.05 \mathrm{e}-9$ & 25 & $-9.89 \mathrm{e}-1$ & $1.49 \mathrm{e}+0$ \\
K_NSold0 & $7.65 \mathrm{e}-7$ & $4.46 \mathrm{e}-7$ & 57 & $-9.92 \mathrm{e}-1$ & $1.49 \mathrm{e}+0$ \\
K_NSold1 & $2.30 \mathrm{e}-7$ & $1.48 \mathrm{e}-8$ & 149 & $-9.92 \mathrm{e}-1$ & $1.49 \mathrm{e}+0$ \\
K_NSold2 & $9.68 \mathrm{e}-7$ & $6.93 \mathrm{e}-7$ & 52 & $-9.82 \mathrm{e}-1$ & $1.48 \mathrm{e}+0$ \\
K_NSold3 & $2.30 \mathrm{e}-7$ & $1.48 \mathrm{e}-8$ & 149 & $-9.92 \mathrm{e}-1$ & $1.49 \mathrm{e}+0$
\end{tabular}

The following Figure 2 demonstrates the efficiency of the above algorithms. We plotted the number of function evaluations for $G^{+}$versus the reached accuracy, where we fixed $T=30$ and $\xi=0.5$. It turned out that K_BrSola and K_NSoli 1

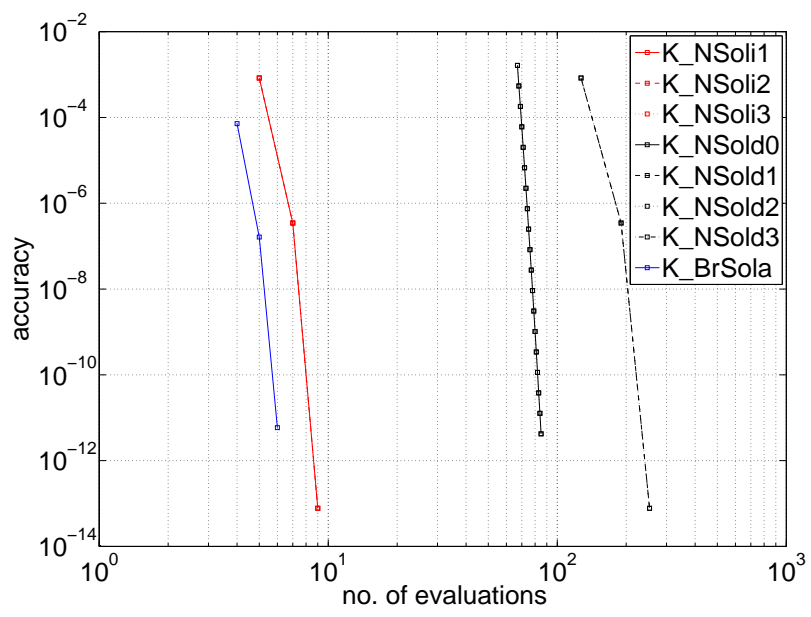

Fig. 2 Efficiency of the Kelley routines in the hyperbolic case

seem to be the most efficient algorithms for this problem, although the first one converges on a smaller interval than the other methods.

\subsubsection{The strongly stable case}

We used different system parameters $a, b$, which together with the error tolerance $\varepsilon$ and constants $\ell, \sigma, \rho, T$ can be found in the following table. In particular, $T>0$ is chosen so that the error is less than $\varepsilon$ on the interval $[-1,1]$. 


\begin{tabular}{ccc|cccc}
$a$ & $b$ & $\varepsilon$ & $\ell$ & $\sigma$ & $\rho$ & $T$ \\
\hline$-1 / 2$ & $9 / 10$ & $10^{-5}$ & $4 / 45$ & $2 / 25$ & 0.020672 & 47
\end{tabular}

As above, the table below shows the performance of the corresponding methods for $\xi_{-}=-0.1, \xi_{+}=0.1, I=[-0.5,0.5]$ and $n=101$.

\begin{tabular}{l|rrrrr} 
method & res & err & eval & $\xi_{\text {min }}$ & $\xi_{\text {max }}$ \\
\hline I_LM & $3.97 \mathrm{e}-6$ & $2.18 \mathrm{e}-6$ & 108 & $-9.91 \mathrm{e}-1$ & $1.50 \mathrm{e}+0$ \\
I_PDL & $3.13 \mathrm{e}-6$ & $1.76 \mathrm{e}-6$ & 108 & $-9.76 \mathrm{e}-1$ & $1.50 \mathrm{e}+0$ \\
K_BrSola & $1.70 \mathrm{e}-6$ & $4.47 \mathrm{e}-8$ & 17 & $-9.15 \mathrm{e}-1$ & $1.26 \mathrm{e}+0$ \\
K_NSoli1 & $8.90 \mathrm{e}-7$ & $2.63 \mathrm{e}-8$ & 23 & $-9.95 \mathrm{e}-1$ & $1.49 \mathrm{e}+0$ \\
K_NSoli2 & $8.90 \mathrm{e}-7$ & $2.62 \mathrm{e}-8$ & 23 & $-9.95 \mathrm{e}-1$ & $1.49 \mathrm{e}+0$ \\
K_NSoli3 & $6.21 \mathrm{e}-7$ & $4.01 \mathrm{e}-8$ & 24 & $-9.95 \mathrm{e}-1$ & $1.49 \mathrm{e}+0$ \\
K_NSoli4 & $7.61 \mathrm{e}-7$ & $2.09 \mathrm{e}-7$ & 25 & $-9.95 \mathrm{e}-1$ & $1.49 \mathrm{e}+0$ \\
K_NSold0 & $9.62 \mathrm{e}-7$ & $7.13 \mathrm{e}-7$ & 130 & $-9.95 \mathrm{e}-1$ & $1.49 \mathrm{e}+0$ \\
K_NSold1 & $2.48 \mathrm{e}-7$ & $6.88 \mathrm{e}-8$ & 318 & $-9.92 \mathrm{e}-1$ & $1.49 \mathrm{e}+0$ \\
K_NSold2 & $8.82 \mathrm{e}-4$ & $9.56 \mathrm{e}-4$ & 106 & $-9.84 \mathrm{e}-1$ & $1.40 \mathrm{e}+0$ \\
K_NSold3 & $2.48 \mathrm{e}-7$ & $6.88 \mathrm{e}-8$ & 318 & $-9.92 \mathrm{e}-1$ & $1.49 \mathrm{e}+0$
\end{tabular}

Compared to the hyperbolic case, the maximal interval of convergence has roughly the same size. However, the results are slightly less accurate. This is underlined by Figure 3 illustrating the efficiency of our algorithms, where we fixed $T=30$. Again, quite solid results have been obtained using K_BrSola and K_NSoli1, although the first mentioned algorithm again converges on a smaller interval.

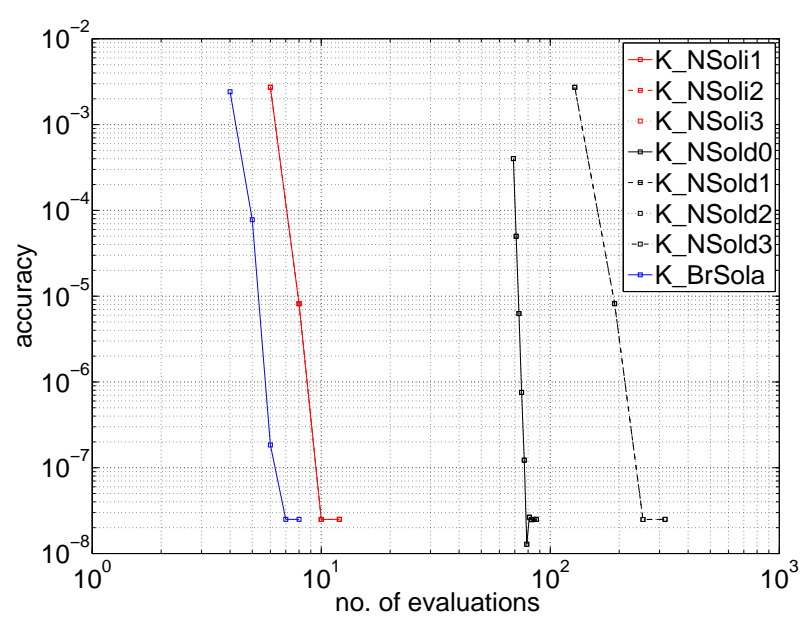

Fig. 3 Efficiency of the Kelley routines in the strongly stable case

\subsubsection{The strongly unstable case}

The system parameters $a, b$, the error tolerance $\varepsilon$ and the constants $\ell, \sigma, \rho, T$ from above are summarized in the following table. Moreover, $T>0$ is chosen to meet the requirement that the error is less than $\varepsilon$ on the interval $[-1,1]$. 


\begin{tabular}{ccc|cccc}
$a$ & $b$ & $\varepsilon$ & $\ell$ & $\sigma$ & $\rho$ & $T$ \\
\hline$-5 / 4$ & 3 & $10^{-5}$ & $7 / 18$ & $7 / 20$ & 0.052731 & 32
\end{tabular}

This strongly unstable situation seems to be the numerically most critical case: The highly unstable character of (21) implies that small inaccuracies in (16) get highly amplified. Indeed, the algorithms K_NSold, K_NSoli and K_BrSola, namely our favorite methods from the previous cases, do not perform very well. Hence, we additionally applied M_DL, M_LM and M_GN, which turn out to be robust but costly. The performance of the more successful algorithms is summarized in the following table with $\xi_{-}=-0.1, \xi_{+}=0.1, I=[-0.5,0.5]$ and $n=101$ :

\begin{tabular}{l|rrrrr} 
method & res & err & eval & $\xi_{\min }$ & $\xi_{\max }$ \\
\hline I_LM & $1.67 \mathrm{e}-2$ & $7.83 \mathrm{e}-3$ & 286 & $-6.47 \mathrm{e}-1$ & $1.24 \mathrm{e}+0$ \\
I_PDL & $1.36 \mathrm{e}-5$ & $8.32 \mathrm{e}-8$ & 294 & $-6.53 \mathrm{e}-1$ & $1.25 \mathrm{e}+0$ \\
M_DL & $4.38 \mathrm{e}-4$ & $1.23 \mathrm{e}-5$ & 3213 & $-6.52 \mathrm{e}-1$ & $1.90 \mathrm{e}+0$ \\
M_LM1 & $1.82 \mathrm{e}-3$ & $9.69 \mathrm{e}-5$ & 4015 & $-6.39 \mathrm{e}-1$ & $1.23 \mathrm{e}+0$ \\
M_GN1 & $3.23 \mathrm{e}-2$ & $3.01 \mathrm{e}-3$ & 5716 & $-6.50 \mathrm{e}-1$ & $1.24 \mathrm{e}+0$ \\
M_GN2 & $4.85 \mathrm{e}-2$ & $6.00 \mathrm{e}-3$ & 6035 & $-6.50 \mathrm{e}-1$ & $1.23 \mathrm{e}+0$
\end{tabular}

Obviously, the numerical amount is drastically larger than in the hyperbolic or strongly stable situation. In order to obtain further performance data, we reduced the length of the Lyapunov-Perron sums to $T=15$ to obtain Figures 4 and 5 illustrating the reached accuracy versus number of iterations. As demonstrated by Figure 4 , the preconditioning strategy for (16) from Remark 8 with damping parameter $\gamma=0.9$ was successful. Unfortunately, preconditioning led to no significant advantages in the hyperbolic and strongly stable case.

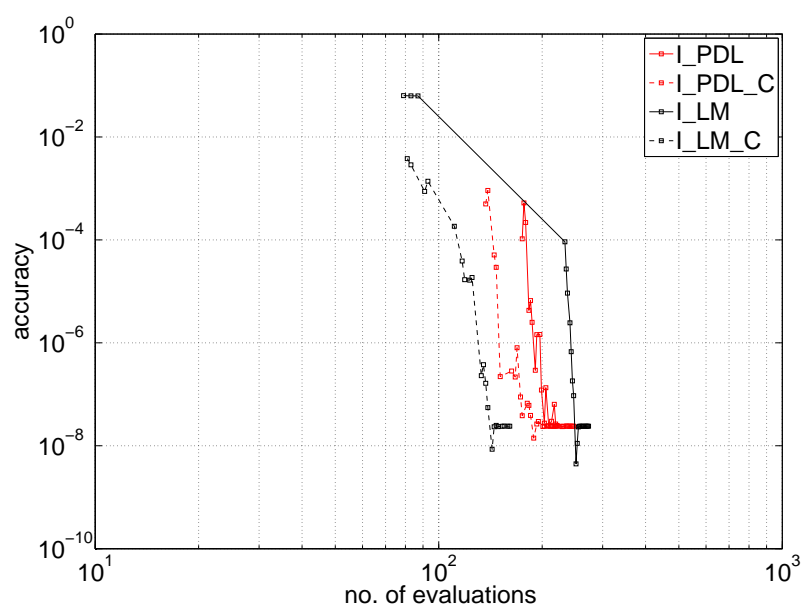

Fig. 4 Efficiency of the IMMOptiBox routines in the strongly unstable case

\subsection{The simplified flour-beetle model}

The biologically motivated model discussed in this subsection is taken from [26] and describes a population of flour beetles. Here, let $a \in(0,1), b>0$ be reals and 


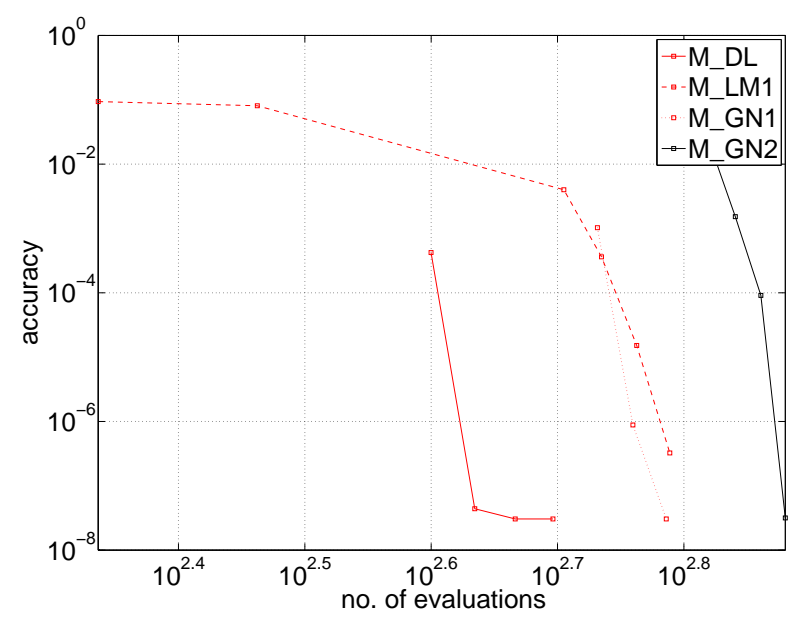

Fig. 5 Efficiency of the MatLab routines in the strongly unstable case

$(\lambda(t))_{t \in \mathbb{I}},(\mu(t))_{t \in \mathbb{I}}$ denote bounded sequences in $[0, \infty)$. We consider the scalar third-order nonautonomous ordinary difference equation

$$
y_{t+3}=a y_{t+2}+b y_{t} e^{-\lambda(t) y_{t+2}-\mu(t) y_{t}},
$$

which is equivalent to the 3 -dimensional first-order system

$$
\left\{\begin{array}{l}
x_{t+1}^{1}=x_{t}^{2} \\
x_{t+1}^{2}=x_{t}^{3} \\
x_{t+1}^{3}=a x_{t}^{3}+b x_{t}^{1} e^{-\lambda(t) x_{t}^{3}-\mu(t) x_{t}^{1}}
\end{array} .\right.
$$

The time-varying coefficients $\lambda(t), \mu(t)$ describe the only significant source of pupal mortality in (22), the adult cannibalism (cf. [26]). For the sake of our analysis, we retreat to the situation $a=\frac{b^{2}-\gamma^{6}}{b \gamma^{2}}$, where $\gamma>0$ is a real number. This implies that the Jacobian of (22) evaluated along the zero solution possesses a pair of complex-conjugated eigenvalues with modulus $\gamma$. To guarantee $a \in(0,1)$ we additionally assume $\gamma \in\left(\sqrt{\omega-\frac{b}{3 \omega}}, \sqrt[3]{b}\right)$ with $\omega:=\sqrt[3]{\frac{b^{2}}{2}+\frac{\sqrt{3 b^{3}(4+27 b)}}{18}}$. The linear transformation $x \mapsto \Lambda x$ with

$$
\Lambda:=\left(\begin{array}{ccc}
\frac{\gamma^{6}-2 b^{2}}{2 b^{2} \gamma^{2}} & \frac{\gamma \sqrt{4 b^{2}-\gamma^{6}}}{2 b^{2}} & 1 \\
-\frac{\gamma^{2}}{2 b} & -\frac{\sqrt{4 b^{2}-\gamma^{6}}}{2 b \gamma} & \frac{b}{\gamma^{2}} \\
1 & 0 & \frac{b^{2}}{\gamma^{4}}
\end{array}\right)
$$

applied to (22) yields a system with decoupled linear part

$$
x_{t+1}=\left(\begin{array}{ccc}
\sigma & \rho & 0 \\
-\rho & \sigma & 0 \\
0 & 0 & \frac{b}{\gamma^{2}}
\end{array}\right) x_{t}+F\left(t, x_{t}\right)
$$


where $\sigma:=-\frac{\gamma^{4}}{2 b}, \rho:=\frac{\gamma \sqrt{4 b^{2}-\gamma^{6}}}{2 b}$, and we have abbreviated $(f$ is the r.h.s. of $(22))$

$$
F(t, x):=\Lambda^{-1} f(t, \Lambda x)-\left(\begin{array}{ccc}
\sigma & \rho & 0 \\
-\rho & \sigma & 0 \\
0 & 0 & \frac{b}{\gamma^{2}}
\end{array}\right) x
$$

It is easy to see that (23) satisfies our assumptions in a neighborhood of 0 , where the dichotomy data is given by $\alpha_{+}=\gamma, \alpha_{-}=\frac{b}{\gamma^{2}}, K_{ \pm}=1$ and $P_{+}:=\left(\begin{array}{lll}1 & & \\ & 1 & \\ & & 0\end{array}\right)$. In order to approximate the invariant fiber bundles $S^{+}$and $S^{-}$of (23) numerically, we fix parameters $b:=\frac{11}{10}, \gamma:=\frac{9}{10}$ (leading to $a=\frac{678559}{891000}$ ) and consider asymptotically constant cannibalism rates $\lambda(t):=1-\frac{1}{\pi} \arctan t, \mu(t):=1+\frac{1}{\pi} \arctan t$. In this particular setting, the cannibalism becomes stationary as $t \rightarrow \pm \infty$, but note that our framework is sufficiently general to capture arbitrary bounded time-dependence. Then the dichotomy rates for (23) are given by $\alpha_{+}=\frac{9}{10}, \alpha_{-}=\frac{110}{81}$. We have computed an approximation of the stable and unstable fiber bundle of (23).

Stable fiber bundle In order to compare the performance of different methods to compute the 2-dimensional stable fiber bundle for (23) (resp. (22)), we introduce the arithmetic mean of the $\ell^{2}$-residual

$$
\operatorname{res}^{+}:=\frac{1}{n_{1} n_{2}} \sum_{i=1}^{n_{1}} \sum_{j=1}^{n_{2}}\left\|G^{+}\left(x, \xi_{i}, \xi_{j}\right)\right\|_{2}
$$

and the number eval ${ }^{+}$of necessary evaluations over a grid of $n_{1} \times n_{2}$ uniformly distributed points $\left(\xi_{i}, \xi_{j}\right)$ in a 2-dimensional box $B$. We fixed $T=15$ and have chosen a tolerance of $\varepsilon=10^{-5}$ in order to approximate the fiber $S^{+}(0)$. In such a situation, for $B=\left[-\frac{1}{4}, \frac{1}{4}\right]^{2}$ and $n_{1}=n_{2}=21$, we arrive at the following table:

\begin{tabular}{l|rrl} 
method & res $^{+}$ & eval $^{+}$ & remark \\
\hline K_BrSola & $1.30 \mathrm{e}-03$ & 39 & line search failures for large $\|\xi\|$ \\
K_NSoli1 & $1.31 \mathrm{e}-06$ & 45 & \\
K_NSoli2 & $1.31 \mathrm{e}-06$ & 45 & \\
K_NSoli3 & $9.02 \mathrm{e}-07$ & 46 & \\
K_NSoli4 & $9.01 \mathrm{e}-07$ & 62 &
\end{tabular}

Note that we have not included the methods I_LM and I_PDL, since they abort due to a too small gradient. Finally, Figure 6 shows the 2-dimensional stable fibers $S^{+}(\tau)$ for $\tau=-10,-2,2,10$ over the square $B$ computed with K_NSoli1.

Unstable fiber bundle We illustrate different methods to get the 1-dimensional unstable fiber bundle of (23) (resp. (22)) using the interval bounds $\xi_{\min }, \xi_{\max }$, the mean residual res ${ }^{-}$and the mean number of evaluations eval ${ }^{-}$. The precise meaning of these factors is analogous to the ones explained in Subsection 4.1.1. The subsequent table shows the performance information for $\xi_{-}=-0.1, \xi_{+}=0.1$, the interval $I=[-0.5,0.5]$ and $n=101$, while computing $S^{-}(0)$. 

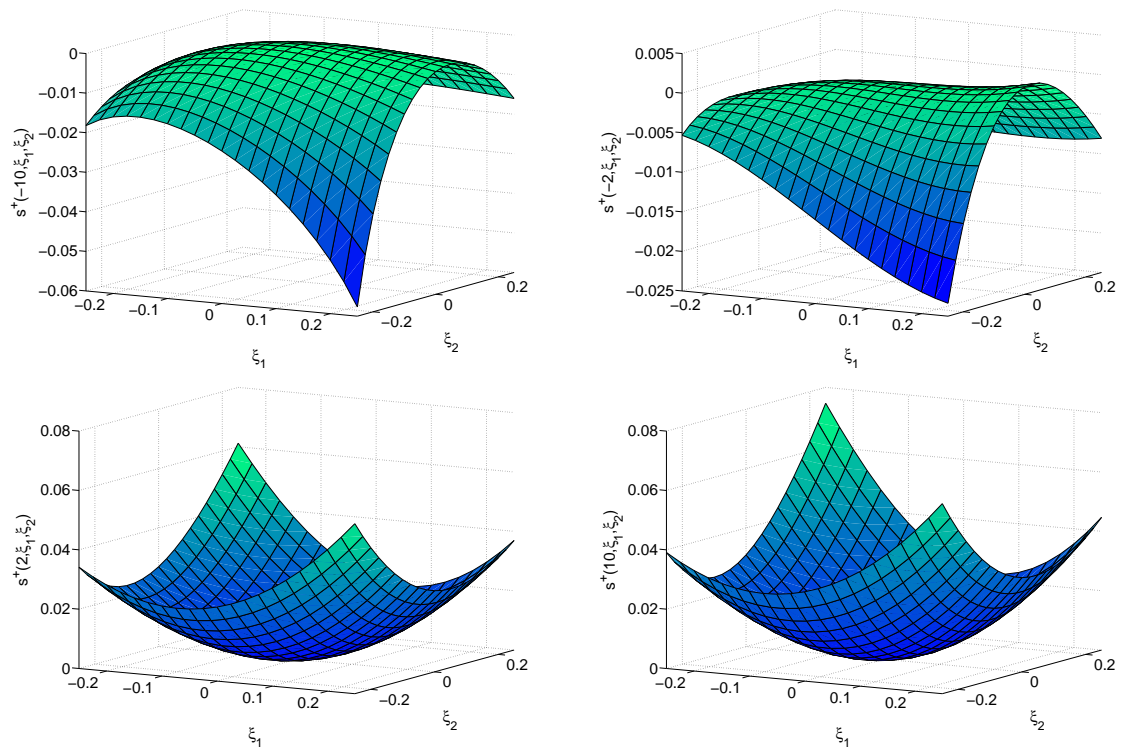

Fig. 6 Stable fibers $S^{+}(\tau)$ in the simplified flour beetle model for $\tau=-10,-2,2,10$

\begin{tabular}{l|rrrrl} 
method & $\mathrm{res}^{-}$ & $\mathrm{eval}^{-}$ & $\xi_{\min }$ & $\xi_{\max }$ & remark \\
\hline I_PDL & $6.21 \mathrm{e}-6$ & 66 & -1.65 & 1.63 & stopped by small $G^{-}$-value \\
K_NSoli1 & $1.69 \mathrm{e}-6$ & 61 & -1.65 & 0.559 & \\
K_NSoli2 & $1.69 \mathrm{e}-6$ & 61 & -1.64 & 0.559 & \\
K_NSoli3 & $1.77 \mathrm{e}-6$ & 108 & -1.65 & 0.547 & \\
K_NSoli4 & $7.77 \mathrm{e}-7$ & 168 & -1.65 & 0.555 &
\end{tabular}

The routine I_LM terminated and $\mathrm{K}_{-} \mathrm{BrSol}$ a aborted due to a line search failure. In Figure 7 we computed and displayed the 1-dimensional unstable fibers $S^{-}(\tau)$ for times $\tau=-10,-2,2$ over the interval $[-1.5,1.5]$ using I_PDL, for which res ${ }^{-}$ is satisfactory small despite error messages due to small $G^{-}$-value. Figure 7 also shows $S^{-}(10)$, where I_PDL converged only on the interval $[-1.5,0.5]$.

We also approximated both the stable and unstable fiber bundle in the simplified flour beetle model with the computer programm GAIO (see [9]). We used the fact that unstable manifolds have pullback attraction properties and that pullback attractors can be approximated via set-oriented techniques (see [10,2] for the theoretical background in the autonomous/nonautonomous context). Our computations approve the results obtained above, however, we made the experience that the computation effort is much higher when using subdivision techniques. This is due to the fact that one needs to study the system on many small boxes iteratively to get a fine covering of the manifold, and mapping boxes means that one has to select many test points in each box. The total amount of evaluations of the right hand side depends on several parameters, and we therefore omit a direct comparison of the two algorithms. Furthermore, note that the approximation of the two-dimensional stable manifold via set-oriented methods was only possible in the small box $[-0.06,0.06]^{3}$, since one needs to approximate the pullback attractor of the system under time reversal which cannot be computed globally. 

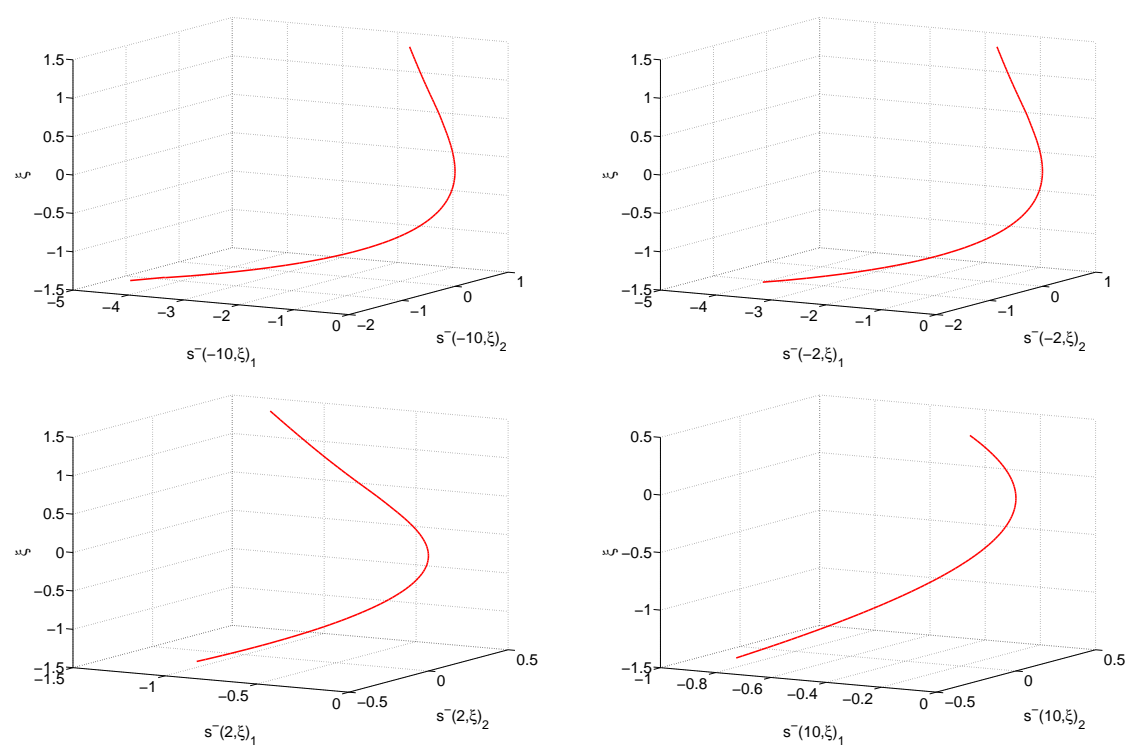

Fig. 7 Unstable fibers $S^{-}(\tau)$ in the simplified flour beetle model for $\tau=-10,-2,2,10$

After all, a (local) Taylor approximation of the stable and center fiber bundles for the simplified flour beetle model can be found in [35].

\subsection{The flour-beetle model}

The difference equation discussed in the previous Subsection 4.2 is indeed a simplification of a more general model. Now we consider the following 3-dimensional nonautonomous system describing a flour beetle population (cf., e.g., [25])

$$
\left\{\begin{array}{l}
x_{t+1}^{1}=b x_{t}^{3} e^{-c_{1}(t) x_{t}^{3}-c_{2}(t) x_{t}^{1}} \\
x_{t+1}^{2}=\left(1-\mu_{1}\right) x_{t}^{1} \\
x_{t+1}^{3}=x_{t}^{2} e^{-c_{3}(t) x_{t}^{3}}+\left(1-\mu_{2}\right) x_{t}^{3}
\end{array}\right.
$$

with model parameters $b>0, \mu_{1}, \mu_{2} \in(0,1)$ and arbitrary bounded sequences $c_{1}, c_{2}, c_{3}: \mathbb{Z} \rightarrow(0, \infty)$. The linearization of (24) in the equilibrium 0 has a real eigenvalue $\rho \in\left(1-\mu_{2}, \infty\right)$ and a complex-conjugated pair $\lambda_{1 / 2}$ satisfying $\left|\lambda_{1 / 2}\right|<\rho$. Hence, the discrete system admits a 2-dimensional pseudo-stable and a 1-dimensional pseudo-unstable fiber bundle.

In numerical calculations we fix parameters $b:=0.65, \mu_{1}:=0.11, \mu_{2}:=0.58$ and choose asymptotically constant cannibalism rates

$$
\begin{aligned}
& c_{1}(k):=0.92+0.45 \arctan (k / 2), \quad c_{2}(k):=0.9+0.13 \arctan (k / 2), \\
& c_{3}(k):=0.18+0.06 \arctan (k / 2) .
\end{aligned}
$$

This yields the eigenvalues $\lambda_{1 / 2}=0.26 \pm 0.67 i$ and $\rho=1$. We fixed $T=15$ and have chosen a tolerance of $\varepsilon=10^{-5}$ in our numerical routines. 
Stable fiber bundle In order to compare the performance of different methods we again computed the arithmetic mean res ${ }^{+}$of the $\ell^{2}$-residual and the number eval ${ }^{+}$ of evaluations over $21 \times 21$ uniformly distributed points in a square $B=[-1,1]^{2}$ :

\begin{tabular}{l|rrl} 
method & res $^{+}$ & eval $^{+}$ & remark \\
\hline I_LM & $8.09 \mathrm{e}-6$ & 74 & aborted by small $G^{+}$-value \\
I_PDL & $1.02 \mathrm{e}-5$ & 68 & aborted by small $G^{+}$-vector \\
K_BrSola & $8.66 \mathrm{e}-6$ & 29 & computed on $22 \times 22$ grid \\
K_NSoli1 & $3.80 \mathrm{e}-6$ & 43 & \\
K_NSoli2 & $3.80 \mathrm{e}-6$ & 43 & \\
K_NSoli3 & $2.55 \mathrm{e}-6$ & 59 & \\
K_NSoli4 & $3.80 \mathrm{e}-6$ & 47 &
\end{tabular}

Figure 8 was created using the routine $\mathrm{K}_{\_}$NSoli1. It shows the 2-dimensional stable fibers $S^{+}(\tau)$ for times $\tau \in\{-10,0,10\}$ over $[-1,1]^{2}$, however not for (24) itself, but for the linearly transformed system admitting a decoupled linearization.
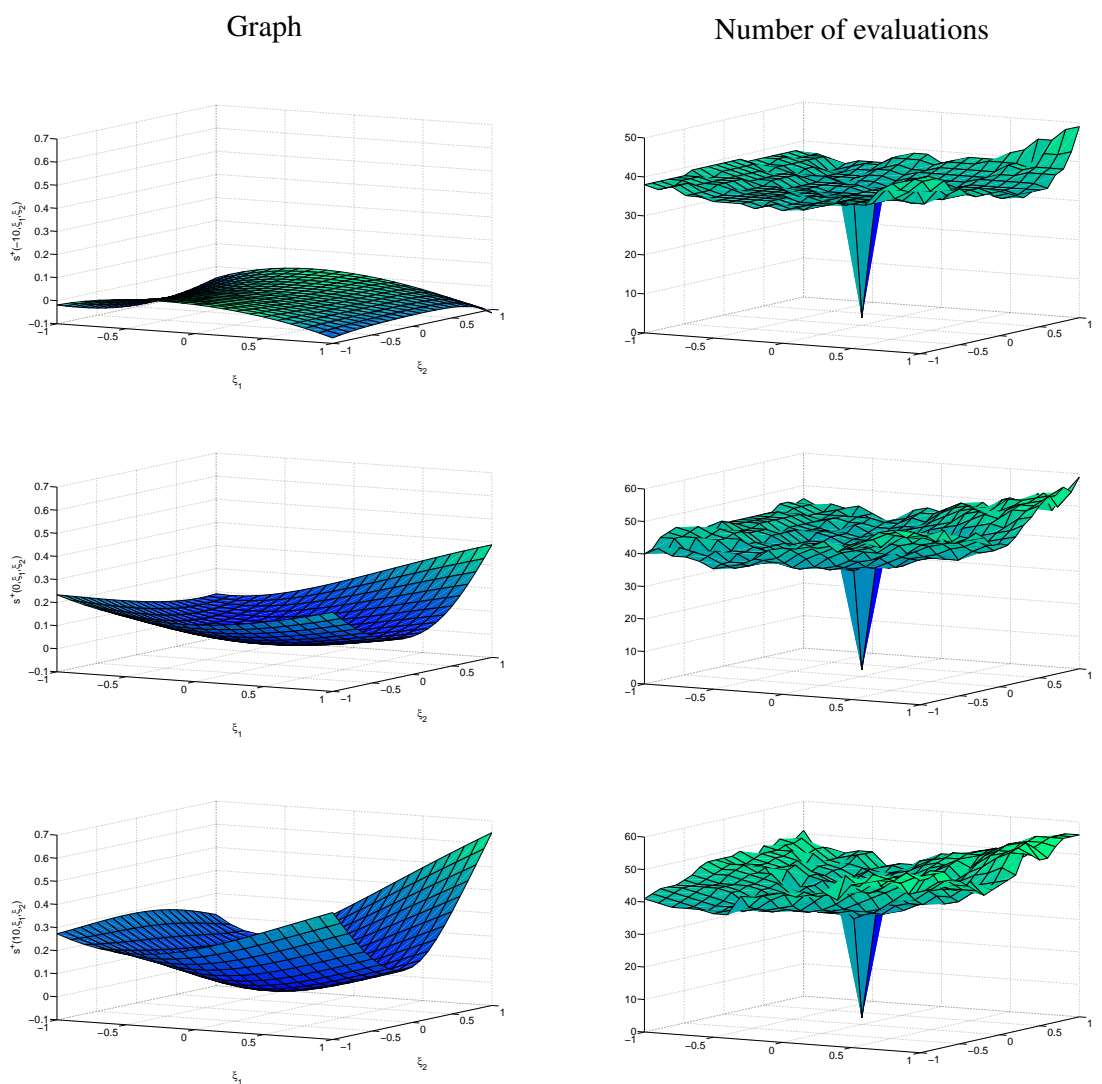

Fig. 8 Stable fibers $S^{+}(\tau)$ (left column) and number of $G^{+}$-evaluations (right column) for times $\tau=-10,0,10$ (f.t.t.d.) 
Center fiber bundle Approximations of the 1-dimensional center fibers $S^{-}(t)$ for $t \in\{-10,0,10\}$ over the interval $[-1,0.1]$ have been computed using K_NSoli 1 and are depicted in Figure 9; again they describe the linearly decoupled version of system (24).

Graph
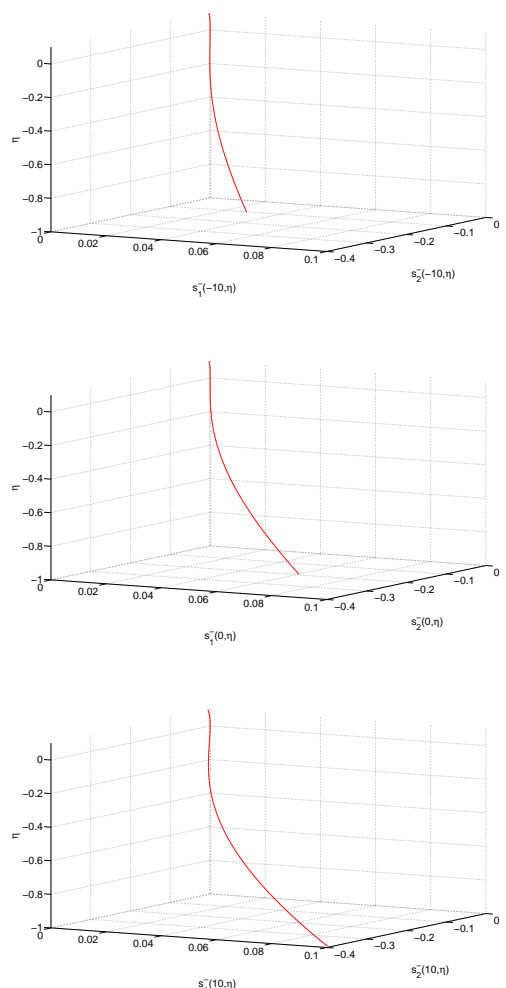

Number of evaluations
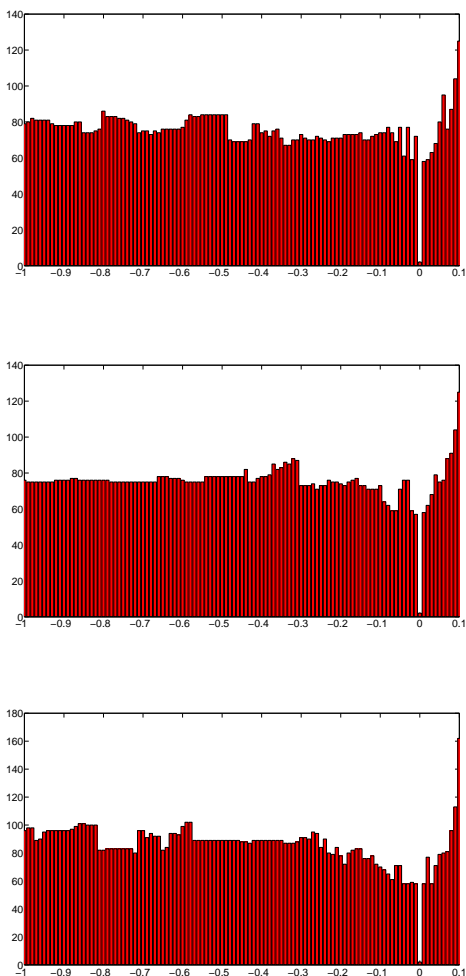

Fig. 9 Center fibers $S^{-}(\tau)$ (left column) and number of $G^{-}$-evaluations (right column) for times $\tau=-10,0,10$ (f.t.t.d.)

\subsection{Hénon map}

In order to illustrate the efficiency of our pseudo-arclength path-following algorithm (Algorithm 3), we computed the unstable manifold $S^{-}$corresponding to the fixed point $\left(x^{*}, y^{*}\right):=\left(\frac{\sqrt{609}}{28}-\frac{1}{4}, \frac{3 \sqrt{609}}{280}-\frac{3}{40}\right) \approx(0.63,0.19)$ of the Hénon system

$$
\left\{\begin{array}{l}
x_{t+1}=y_{t}+1-a x_{t}^{2}, \\
y_{t+1}=b x_{t}
\end{array}\right.
$$


for the typically used parameters $a=1.4$ and $b=0.3$. We chose $\varepsilon=10^{-6}, T=20$, $h_{\min }=2^{-15} h_{\max }$, the inital step-size $h=10^{-4}$ and tested different algorithms to solve the corresponding nonlinear systems. Their performance can be compared using the averaged number of evaluations $\overline{\text { eval }}=\frac{\text { eval }}{s_{\max }-s_{\min }}$ and the corresponding averaged number of necessary step-size corrections $\overline{c o r r}=\frac{\text { corr }}{s_{\max }-s_{\min }}$, respectively.

\begin{tabular}{lr|rrrr} 
method & $h_{\max }$ & $s_{\min }$ & $s_{\max }$ & $\overline{\text { eval }}$ & $\overline{\text { corr }}$ \\
\hline K_NSoli1 & $2.5 \mathrm{e}-3$ & -123.5 & 74.39 & 35901 & 0.1668 \\
M_DL & $2.5 \mathrm{e}-3$ & -59.3 & 8.74 & 92775 & 64.21 \\
\hline M_LM1 & $2.5 \mathrm{e}-3$ & -125.0 & 125.0 & 54806 & 0 \\
& $5.0 \mathrm{e}-3$ & -249.8 & 245.0 & 27421 & 0.068 \\
& $1.0 \mathrm{e}-2$ & -499.5 & 499.9 & 15420 & 0.044 \\
& $5.0 \mathrm{e}-2$ & -2496.3 & 2485.1 & 3806 & 0.062
\end{tabular}

The Levenberg-Marquardt method M_LM1 proves to be very robust and needed few step-size adaptions. While the other methods aborted due to $h<h_{\min }$ we interrupted M_LM1 after 50000 successful increments of the continuation parameter, while the other algorithms reacted more sensitive to larger upper bounds for $h$.

The results using M_LM1 are visualized in the left column of Figure 10. For comparison reasons, in the right column we also approximated the Hénon attractor using rigerous set-oriented numerics in form of the program GAIO (cf. [9]). The unstable manifold $S^{-}$, as embedded manifold, is contained in the strange attractor $A$ of the Hénon map, i.e., $S^{-} \subseteq A$. GAIO yields a covering of the attractor, which can be made arbitrarily fine, and therefore yields an outer approximation of the unstable manifold. Our continuation approach provides more insight into the fine structure of the manifold, but is only able to compute a part of $S^{-}$and consequently delivers a somewhat incomplete picture. Nevertheless, as demonstrated by Figure 10 at least on a graphical level our approximation is quite accurate.

To provide some further details on our computation using GAIO, we started with $2^{13}=8192$ boxes and every step contains 4 continuation steps, before subdivision had been applied. Each box required 100 randomly chosen points in which the Hénon map was evaluated. This led to 1.562.126.800 Hénon evaluations.

\subsection{Colpitts oscillator}

The following example illustrates our methods applied to an autonomous ODE, whose right-hand side is globally Lipschitz but not continuously differentiable. The Colpitts oscillator (see, for instance, [28]) is one of the most widely used single-transistor circuits to produce sinusoidal oscillations. Mathematically, it is given by a 3-dimensional system

$$
\left\{\begin{array}{l}
\dot{x}^{1}=\frac{1}{\vartheta}\left(x^{3}-n\left(x^{2}\right)\right) \\
\dot{x}^{2}=\frac{k-1}{\vartheta} x^{2} \\
\dot{x}^{3}=-\frac{\vartheta}{k}\left(x^{1}-x^{2}\right)-2 q x^{3}
\end{array}\right.
$$

with parameters $\vartheta, q>0, k>1$ and a piecewise-defined nonlinearity

$$
n(x):=\left\{\begin{array}{ll}
-x, & x \leq 1 \\
-1, & x>1
\end{array} .\right.
$$



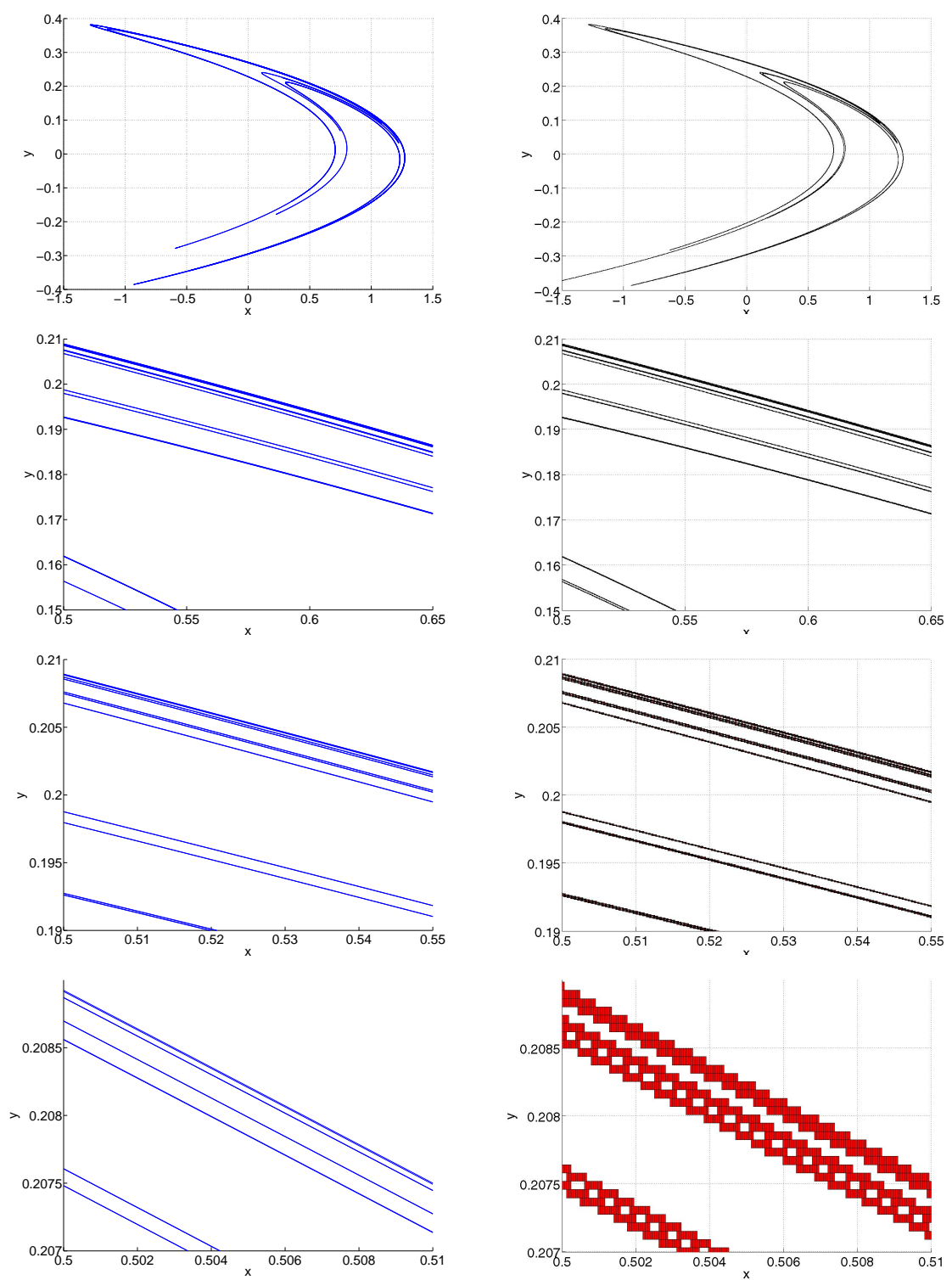

Fig. 10 Magnifications of the Hénon attractor: Unstable manifold of the Hénon map computed using Algorithm 3 (left column) versus the attractor computed with GAIO (right column)

Obviously, equation (25) admits the trivial solution, and we can write it in semilinear form $\dot{x}=A x+F(x)$ with mappings

$$
A:=\left(\begin{array}{ccc}
0 & 0 & \frac{1}{\vartheta} \\
0 & 0 & \frac{k-1}{\vartheta} \\
-\frac{\vartheta}{k} & -\frac{\vartheta}{k} & -2 q
\end{array}\right), \quad F(x):=-\frac{1}{\vartheta}\left(\begin{array}{c}
n\left(x_{2}\right) \\
0 \\
0
\end{array}\right) .
$$


Since the spectrum of $A$ is given by $\sigma(A)=\{0,-q \pm \omega\}, \omega:=\sqrt{q^{2}-1}$, we can verify that (25) possesses a 2 -dimensional stable and a 1 -dimensional center manifold. The computation of these manifolds get simplified, if we transform (25) via

$$
\Lambda:=\left(\begin{array}{ccc}
1 & 1 & -1 \\
k-1 & k-1 & 1 \\
\vartheta(-q+\omega) & \vartheta(-q-\omega) & 0
\end{array}\right)
$$

and arrive at the decoupled system

$$
\dot{x}=\left(\begin{array}{ccc}
-q-\omega & & \\
& -q+\omega & \\
& & 0
\end{array}\right) x-\frac{n\left((k-1)\left(x^{1}+x^{2}\right)+x^{3}\right)}{k \vartheta}\left(\begin{array}{c}
\frac{q+\omega}{2 \omega} \\
\frac{-q+\omega}{2 \omega} \\
1-k
\end{array}\right) \text {. }
$$

As suggested in Subsection 3.3, we apply a numerical one-step scheme to the autonomous ODE (25). For simplicity reasons we restrict to the explicit Euler method for step-size $h=0.01$ and use Algorithm 1 to the resulting difference equation. We fixed parameter values $q=2, \vartheta=8, k=5$ leading to a 2-dimensional stable and a 1-dimensional center manifold.

The invariant manifolds of this temporal Euler discretization have been computed with $T=15$ and accuracy $\varepsilon=10^{-5}$. For the stable manifold $S^{+}$we used a grid of $21 \times 21$ uniformly distributed points in the box $B=[-1,1]^{2}$. Our different methods led to the following results:

\begin{tabular}{l|rrl} 
method & res $^{+}$ & eval $^{+}$ & remark \\
\hline I_LM & $5.75 \mathrm{e}-5$ & 65 & aborted by small $G^{+}$-vector \\
I_PDL & $1.12 \mathrm{e}-6$ & 60 & aborted by small $G^{+}$-vector \\
K_BrSola & $8.44 \mathrm{e}-6$ & 30 & line search failures \\
K_NSoli1 & $2.70 \mathrm{e}-6$ & 56 & \\
K_NSoli2 & $2.70 \mathrm{e}-6$ & 56 & \\
K_NSoli3 & $1.34 \mathrm{e}-6$ & 152 & \\
K_NSoli4 & $2.56 \mathrm{e}-6$ & 57 &
\end{tabular}

Concerning the center manifold $S^{-}$, for several methods, we computed the arithmetic mean of the $\ell^{2}$-residual and the number of evaluations over the interval $I=[-2,2]$ using $n=101$ intermediate points:

\begin{tabular}{l|rrl} 
method & res $^{-}$ & eval $^{-}$ & remark \\
\hline I_LM & $6.77 \mathrm{e}-5$ & 64 & stopped by small $G^{-}$-vector \\
I_PDL & $1.30 \mathrm{e}-6$ & 57 & stopped by small $G^{-}$-vector \\
K_NSoli1 & $2.91 \mathrm{e}-6$ & 54 & \\
K_NSoli2 & $2.91 \mathrm{e}-6$ & 54 & \\
K_NSoli3 & $3.42 \mathrm{e}-6$ & 85 & \\
M_DL & $7.93 \mathrm{e}-10$ & 180 &
\end{tabular}

Unfortunately, we obtained no convergence for $\mathrm{K} \_\mathrm{BrSola}$ (line search failures) and K_NSoli4. The numerical approximations using K_NSoli 1 for the stable manifold $S^{+}$and the center manifold $S^{-}$are illustrated in Figure 11. 

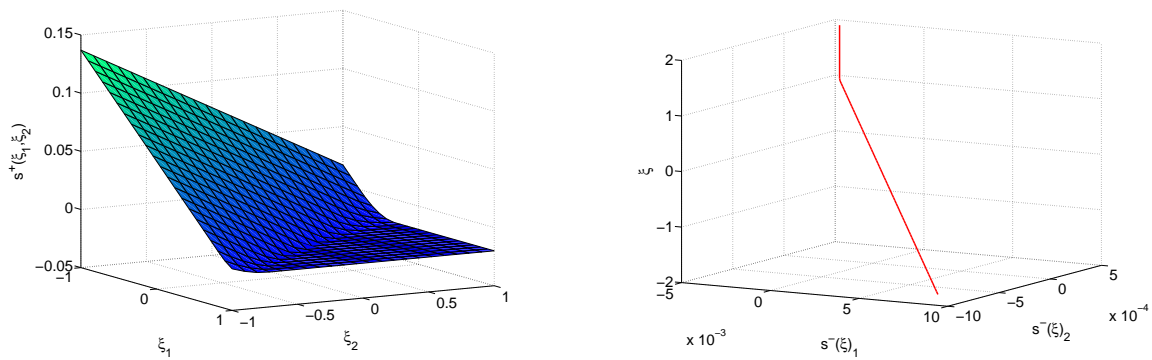

Fig. 11 Stable manifold (left) and center manifold (right) for the forward Euler discretization of the Colpitts oscillator (26)

\subsection{Discretized reaction-diffusion equations}

In this final more comprehensive example, we are interested in approximating the inertial manifold of a scalar nonautonomous reaction-diffusion equation. Thereto, we apply our results to a finite-dimensional difference equation, which has been obtained from the original evolutionary PDE by a Bubnov-Galerkin method for spatial, and a linearly implicit Euler scheme for temporal discretization. Error estimates for such full discretizations have been obtained in [19].

We study the following nonautonomous reaction-diffusion equation

$$
\partial_{t} u=\partial_{x x} u-f(t, u),
$$

subject to homogeneous Dirichlet boundary conditions $u(t, 0)=u(t, \pi)=0$ and an initial condition $u(\tau, x)=u_{0}(x)$ for given data $\tau \in \mathbb{R}, u_{0} \in L^{2}(0, \pi)$.

This problem fits into the framework of [8], provided we also suppose that $f: \mathbb{R} \times \mathbb{R} \rightarrow \mathbb{R}$ is continuous, that the partial derivative $\partial_{2}^{2} f: \mathbb{R} \times \mathbb{R} \rightarrow \mathbb{R}$ exists as a continuous function and that there exist reals $C_{1}, C_{2}, C_{3}, \gamma>0, p \geq 2$ such that

$$
\gamma|v|^{p}-C_{1} \leq f(t, v) v, \quad|f(t, v)|^{\frac{p}{p-1}} \leq C_{2}\left(1+|v|^{p}\right), \quad-C_{3} \leq \partial_{2} f(t, v)
$$

for all $t, v \in \mathbb{R}$ and provided we can choose $K_{1}, K_{2}:[0, \infty) \rightarrow \mathbb{R}$ such that

$$
K_{i}(r) \geq \sup _{t \in \mathbb{R}} \sup _{|v| \leq \sqrt{\pi} r}\left|\partial_{2}^{i} f(t, v)\right| \quad \text { for all } i=1,2, r \geq 0 .
$$

Using a Galerkin technique, it is shown in [8, p. 114, Proposition 2.1] that the nonautonomous equation (27) generates a dissipative 2-parameter semiflow on the space $L^{2}(0, \pi)$ - note that we write $L^{2}$ instead of $L^{2}(0, \pi)$ from now on, and proceed similarly with the spaces $H_{0}^{1}$ or $C^{0}$.

On the other hand, following [38, Section 5.1], we can formulate (27) as abstract nonautonomous evolutionary equation

$$
\dot{u}+L u=g(t, u)
$$

with linear part $L:=-\partial_{x x}$ and substitution operator $g(t, u)(x):=-f(t, u(x))$. Then, referring to [38, p. 272, Theorem 51.1], the mild solutions of (30) generate a dissipative 2-parameter semigroup on $H_{0}^{1}$, and in [8, p. 290, Proposition 3.5] 
it is shown that the radius of the corresponding absorbing set in $H_{0}^{1}$ is bounded by

$$
r_{0}:=2 \sqrt{2 C_{1} C_{3}} .
$$

It is known that the eigenvalues of the negative Laplacian $L$ with zero boundary condition $u(0)=u(\pi)=0$ are given by $\lambda_{n}=n^{2}, n \in \mathbb{N}$, with corresponding pairwise $L^{2}$-orthonormal eigenfunctions

$$
e_{n}(x)=\sqrt{\frac{2}{\pi}} \sin (n x) \text { for all } n \in \mathbb{N} .
$$

In addition, let $P_{d}: L^{2} \rightarrow L^{2}$ be the orthogonal projection onto the $d$-dimensional space $\operatorname{span}\left\{e_{1}, \ldots, e_{d}\right\}$ and $Q_{d}:=I-P_{d}$ be the complementary projector.

After these preparations we prove that (27) admits an inertial manifold:

Proposition 4 Assume that (28), (29) hold and the integer $d \geq 0$ satisfies

$$
d>\sqrt{2} L\left(r_{0}\right)-1 / 2, \quad L(r):=\sqrt{2 K_{1}(r)^{2}+r^{2} K_{2}(r)^{2}} .
$$

Then the reaction-diffusion equation (27) has a d-dimensional inertial manifold

$$
S^{-}=\left\{\left(\tau, \xi+s^{-}(\tau, \xi)\right) \in \mathbb{R} \times H_{0}^{1}: \xi \in \operatorname{im} P_{d}\right\}
$$

with a smooth function $s^{-}: \mathbb{R} \times \operatorname{im} P_{d} \rightarrow \operatorname{im} Q_{d}$.

Proof In order to employ a suitable inertial manifold theorem, some preparations are due. As in [38, p. 271] one shows that $g: \mathbb{R} \times H_{0}^{1} \rightarrow H_{0}^{1}$ is well-defined and satisfies the local Lipschitz condition

$$
\|g(t, u)-g(t, \bar{u})\|_{H_{0}^{1}} \leq L(r)\|u-\bar{u}\|_{H_{0}^{1}} \quad \text { for all } t \in \mathbb{R}, u, \bar{u} \in \bar{B}_{r}(0) \subseteq H_{0}^{1}
$$

and $n \in \mathbb{N}$. Note that the explicit form of the constant $L(r)$ given above results from the compact embedding $H_{0}^{1} \hookrightarrow C^{0}$ (cf., e.g., [8, p. 30, Theorem 1.2]) satisfying an explicit estimate $\|u\|_{C^{0}} \leq \sqrt{\pi}\|u\|_{H_{0}^{1}}$ for all $u \in H_{0}^{1}$. Using an appropriate retraction mapping, it is possible to modify $g(t, \cdot)$ outside the absorbing ball $B_{r_{0}}(0)$ such that the altered nonlinearity $\bar{g}$ is globally bounded and satisfies a global Lipschitz condition in the second argument with constant $L\left(r_{0}\right)$ (uniformly in $t \in \mathbb{R}$ ). Hence, we can apply [23, Corollary 4.1] to establish the existence of the desired inertial manifold, since

$$
\|\bar{g}(t, u)-\bar{g}(t, \bar{u})\|_{H_{0}^{1}} \leq \sqrt{2} L(r) \max \left\{\left\|P_{n}[u-\bar{u}]\right\|_{H_{0}^{1}},\left\|Q_{n}[u-\bar{u}]\right\|_{H_{0}^{1}}\right\} .
$$

In our situation, the spectral gap condition in [23, p. 934] reduces to the estimate $2 \sqrt{2} L\left(r_{0}\right)<\lambda_{n+1}-\lambda_{n}=2 n+1$, and this implies our claim.

Now we are in a position to describe our discretization strategy for (27). First, the Bubnov-Galerkin approximation with $N$ Fourier modes, $N \geq 1$, is obtained by inserting the ansatz

$$
u(t, x)=\sum_{i=1}^{N} v_{i}(t) e_{i}(x)
$$


into (27) and taking the $L^{2}$-inner product with $e_{j}, j \in[1, N]_{\mathbb{Z}}$, leads to an initial value problem in the space $\operatorname{im} P_{N}$. We canonically identify this linear space with $\mathbb{R}^{N}$ and arrive at the $N$-dimensional ODE

$$
\dot{v}_{j}=-j^{2} v_{j}+f_{j}(t, v) \quad \text { for all } j \in[1, N]_{\mathbb{Z}}
$$

with the nonlinearities $f_{j}: \mathbb{R} \times \mathbb{R}^{N} \rightarrow \mathbb{R}$,

$$
f_{j}(t, v)=-\int_{0}^{\pi} f\left(t, \sum_{i=1}^{N} v_{i}(t) e_{i}(x)\right) e_{j}(x) d x
$$

and initial condition $v(\tau)=\eta, \eta_{j}=\int_{0}^{\pi} u_{0}(x) e_{j}(x) d x$. Respecting the (mild) stiffness of the matrix $-\operatorname{diag}\left(j^{2}\right)_{j=1}^{N}$, we apply a linearly implicit Euler discretization (with step-size $h>0$ ) to (32) and arrive at the nonautonomous difference equation

$$
v_{t+1}=A_{h} v_{t}+F_{h}\left(t, v_{t}\right)
$$

with linear part $A_{h}:=\operatorname{diag}\left(\frac{1}{1+h j^{2}}\right)_{j=1}^{N}$ and a nonlinearity $F_{h}: \mathbb{Z} \times \mathbb{R}^{N} \rightarrow \mathbb{R}^{N}$, whose components are given by

$$
F_{h}(t, v)_{j}:=\frac{h}{1+h j^{2}} f_{j}(\tau+h t, v) \quad \text { for all } j \in[1, N]_{\mathbb{Z}} .
$$

Henceforth, we deduce the existence of an attractive invariant fiber bundle for the difference equation (34). Choosing an integer $d$ according to (31), the linear part of (34) satisfies Hypothesis 1 with dichotomy data $K_{-}=K_{+}=1$,

$$
\alpha_{+}:=\left(1+h(d+1)^{2}\right)^{-1}, \quad \alpha_{-}:=\left(1+h d^{2}\right)^{-1} .
$$

and the invariant projector $P_{-}=\operatorname{diag}(1, \ldots, 1,0, \ldots, 0)$. Moreover, it is possible to verify Hypothesis 2. Thus, we can employ the methods from Section 3 to approximate the invariant fiber bundle

$$
S_{h, N}^{-}=\left\{\left(k, \xi+s_{h, N}^{-}(k h, \xi)\right) \in \mathbb{Z} \times \mathbb{R}^{N}: k \in \mathbb{Z}, \xi \in \operatorname{im} P_{-}\right\}
$$

of the discretization (34). An error estimate relating the nonautonomous inertial manifold $S^{-}$of the full reaction diffusion equation (27) (cf. Proposition 4) to the finite-dimensional invariant fiber bundles $S_{h, N}^{-}$, can be found in [19, Theorem 5.3] and is of the form

$$
\left\|s_{h, N}^{-}(h k, \xi)-s^{-}(h k, \xi)\right\| \leq K_{1} \lambda_{N} h+K_{2} \sqrt{\frac{\lambda_{d+1}}{\lambda_{N+1}}}
$$

with constants $K_{1}, K_{2}>0$, sufficiently large $N$ and small $h$.

In the remaining part of the paper, it is our intention to compute fibers of the nonautonomous set $S_{h, N}^{-}$. Here we retreat to a scalar Chafee-Infante equation with time-dependent coefficients

$$
\partial_{t} u=\partial_{x x} u+\lambda(t) u-\mu(t) u^{3},
$$


under the above initial boundary conditions. For continuous bounded functions $\mu, \lambda: \mathbb{R} \rightarrow(0, \infty)$ is not difficult to show that (36) fulfills assumption (28) with nonlinearity $f(t, v)=\mu(t) v^{3}-\lambda(t) v$ and $p=4$, provided there exists a $\gamma>0$ with $\gamma<\mu(t)$ for all $t \in \mathbb{R}$ and

$$
C_{1}:=\sup _{t \in \mathbb{R}} \frac{\lambda(t)^{2}}{4(\mu(t)-\gamma)}, \quad C_{3}:=\sup _{t \in \mathbb{R}} \lambda(t)<\infty .
$$

Furthermore, we can choose the functions $K_{1}, K_{2}:[0, \infty) \rightarrow \mathbb{R}$ from (29) as

$$
K_{1}(r):=\max \left\{C_{3}, 3 \pi r^{2} \sup _{t \in \mathbb{R}} \mu(t)-\inf _{t \in \mathbb{R}} \lambda(t)\right\}, \quad K_{2}(r):=6 \sqrt{\pi} r \sup _{t \in \mathbb{R}} \mu(t) .
$$

In the next step we compute a Galerkin approximation for (36). Unfortunately, the constants $K_{1}, K_{2}>0$ in the mentioned error estimate (35) from [19, Theorem 5.3] are not immediately accessible. For this reason, we heuristically choose a spatial approximation of order $N=6$. With help of some computer algebra to evaluate the integrals (33), the resulting nonlinearities $f_{1}, \ldots, f_{6}$ read as follows:

$$
\begin{aligned}
& f_{1}(t, v)=\frac{\mu(t)}{2 \pi}\left(-6 v_{2} v_{3} v_{4}+3 v_{2}^{2} v_{5}-6 v_{1} v_{5}^{2}-6 v_{1} v_{6}^{2}-6 v_{2} v_{4} v_{5}-6 v_{1} v_{3}^{2}\right. \\
& -3 v_{3}^{2} v_{5}+6 v_{1} v_{3} v_{5}-3 v_{2}^{2} v_{3}+6 v_{2} v_{3} v_{6}+6 v_{1} v_{4} v_{6}-6 v_{2} v_{5} v_{6} \\
& \left.-6 v_{1} v_{4}^{2}-6 v_{1} v_{2}^{2}+6 v_{1} v_{2} v_{4}-3 v_{1}^{3}-6 v_{3} v_{4} v_{6}+3 v_{1}^{2} v_{3}\right)+\lambda(t) v_{1}, \\
& f_{2}(t, v)=\frac{\mu(t)}{2 \pi}\left(-6 v_{3} v_{4} v_{5}-6 v_{1} v_{2} v_{3}+3 v_{1}^{2} v_{4}+6 v_{1} v_{3} v_{6}-6 v_{1} v_{4} v_{5}-3 v_{2}^{3}\right. \\
& -6 v_{1}^{2} v_{2}-6 v_{1} v_{5} v_{6}-3 v_{4}^{2} v_{6}-6 v_{1} v_{3} v_{4}-3 v_{3}^{2} v_{4}-6 v_{2} v_{4}^{2} \\
& \left.+3 v_{2}^{2} v_{6}-6 v_{2} v_{6}^{2}-6 v_{3} v_{5} v_{6}-6 v_{2} v_{5}^{2}+6 v_{1} v_{2} v_{5}-6 v_{2} v_{3}^{2}\right)+\lambda(t) v_{2}, \\
& f_{3}(t, v)=\frac{\mu(t)}{2 \pi}\left(v_{1}^{3}-3 v_{3}^{3}-6 v_{2} v_{5} v_{6}-3 v_{1} v_{2}^{2}-6 v_{1} v_{3} v_{5}-6 v_{1} v_{2} v_{4}\right. \\
& +6 v_{1} v_{2} v_{6}-6 v_{1} v_{4} v_{6}-6 v_{2} v_{4} v_{5}-6 v_{4} v_{5} v_{6}-6 v_{2} v_{3} v_{4}-6 v_{3} v_{4}^{2} \\
& \left.+3 v_{1}^{2} v_{5}-6 v_{3} v_{5}^{2}-6 v_{3} v_{6}^{2}-6 v_{1}^{2} v_{3}-3 v_{4}^{2} v_{5}-6 v_{2}^{2} v_{3}\right)+\lambda(t) v_{3}, \\
& f_{4}(t, v)=\frac{\mu(t)}{2 \pi}\left(-6 v_{3} v_{4} v_{5}-3 v_{2} v_{3}^{2}-6 v_{4} v_{5}^{2}-3 v_{4}^{3}-6 v_{2}^{2} v_{4}-6 v_{2} v_{4} v_{6}\right. \\
& +3 v_{1}^{2} v_{6}-6 v_{3}^{2} v_{4}-6 v_{1} v_{3} v_{6}+3 v_{1}^{2} v_{2}-6 v_{1} v_{2} v_{3}-6 v_{3} v_{5} v_{6} \\
& \left.-6 v_{1} v_{2} v_{5}-6 v_{2} v_{3} v_{5}-6 v_{1}^{2} v_{4}-3 v_{5}^{2} v_{6}-6 v_{4} v_{6}^{2}\right)+\lambda(t) v_{4}, \\
& f_{5}(t, v)=\frac{\mu(t)}{2 \pi}\left(-6 v_{1} v_{2} v_{6}-6 v_{4} v_{5} v_{6}-3 v_{3} v_{4}^{2}-6 v_{4}^{2} v_{5}-3 v_{1} v_{3}^{2}+3 v_{1}^{2} v_{3}\right. \\
& -6 v_{1} v_{2} v_{4}-6 v_{3} v_{4} v_{6}+3 v_{1} v_{2}^{2}-6 v_{2}^{2} v_{5}-6 v_{2} v_{3} v_{6}-6 v_{5} v_{6}^{2} \\
& \left.-6 v_{2} v_{3} v_{4}-6 v_{1}^{2} v_{5}-3 v_{5}^{3}-6 v_{3}^{2} v_{5}\right)+\lambda(t) v_{5}, \\
& f_{6}(t, v)=\frac{\mu(t)}{2 \pi}\left(-6 v_{1} v_{3} v_{4}-3 v_{6}^{3}-3 v_{4} v_{5}^{2}+6 v_{1} v_{2} v_{3}-3 v_{2} v_{4}^{2}-6 v_{2}^{2} v_{6}+v_{2}^{3}\right. \\
& +3 v_{1}^{2} v_{4}-6 v_{5}^{2} v_{6}-6 v_{1} v_{2} v_{5}-6 v_{1}^{2} v_{6}-6 v_{3}^{2} v_{6}-6 v_{4}^{2} v_{6} \\
& \left.-6 v_{3} v_{4} v_{5}-6 v_{2} v_{3} v_{5}\right)+\lambda(t) v_{6} \text {. }
\end{aligned}
$$

To perform actual computations, we choose $\delta>0$ and define $\mu, \lambda: \mathbb{R} \rightarrow \mathbb{R}$ by

$$
\mu(t): \equiv \delta, \quad \lambda(t):=\delta\left(\frac{\pi}{2}+\arctan t\right) .
$$


Hence, for $\gamma:=\delta / 2$ the radius of the absorbing set for the reaction-diffusion equation (36) is bounded above by $r_{0}=2 \pi^{5 / 2} \delta$. Consequently, Proposition 4 guarantees that (36) admits a nonautonomous inertial manifold $S^{-}$, whose dimension is the minimal integer $d \geq 0$ satisfying

$$
d>2 \pi \delta \sqrt{\max \left\{12 \pi^{3} \delta^{2}, 1\right\}^{2}+288 \pi^{5} \delta^{4}}-1 / 2 .
$$

We are fixing the parameter value $\delta=\frac{471}{5000}$ and the evolutionary equation (27) admits a 2-dimensional inertial manifold, i.e., we can choose $d=2$ and also obtain a 2-dimensional invariant fiber bundle $S_{h, 6}^{-}$for the Bubnov-Galerkin-Euler discretization (34). In particular, this nonautonomous set $S_{h, 6}^{-}$is given as graph of a function $s_{h, 6}^{-}: \mathbb{Z} \times \mathbb{R}^{2} \rightarrow \mathbb{R}^{4}$.

We used Algorithm 1 to approximate the function $s_{h, 6}^{-}$over the square $[-1,1]^{2}$ using a uniform grid of $21 \times 21$ points, for an Euler step-size $h=0.1, T=15$ and accuracy $\varepsilon=10^{-5}$. The corresponding performance data for the method K_NSoli 1 are displayed in the following table:

\begin{tabular}{r|ccccc}
$\tau$ & -20 & -10 & 0 & 10 & 20 \\
\hline err & $3.58 \mathrm{e}-6$ & $3.49 \mathrm{e}-6$ & $5.72 \mathrm{e}-4$ & $4.84 \mathrm{e}-3$ & $5.13 \mathrm{e}-3$ \\
eval & 645 & 654 & 696 & 856 & 865
\end{tabular}

It is apparent that computing fibers $S_{h, 6}^{-}(\tau)$ becomes more expensive and less accurate as the fiber index $\tau$ grows. The results of this computation are visualized in Figure 12 showing the components of $s_{h, 6}^{-}(\tau, \xi)$ for a fixed fiber with $\tau=0$. In addition, Figure 13 illustrates how the first component $s_{h, 6}^{-}(\tau, \xi)_{1}$ changes under varying fibers with $\tau=-20,20$.
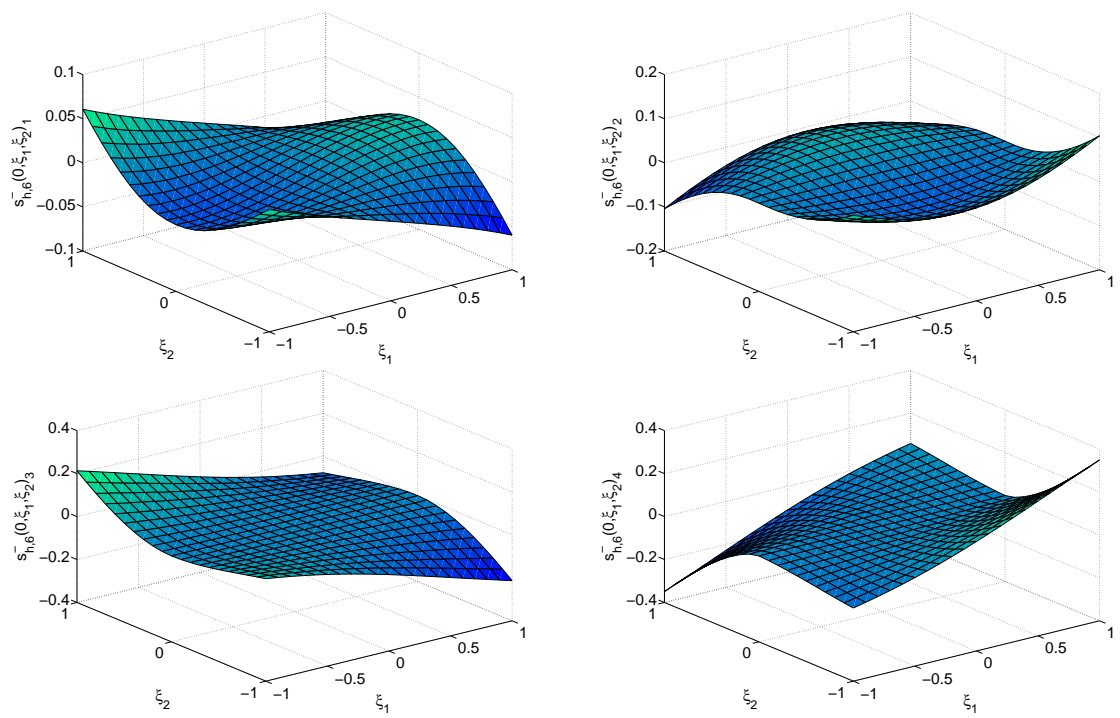

Fig. 12 Graphs of $s_{h, 6}^{-}\left(0, \xi_{1}, \xi_{2}\right)_{i}$ for $i=1,2,3,4$ 

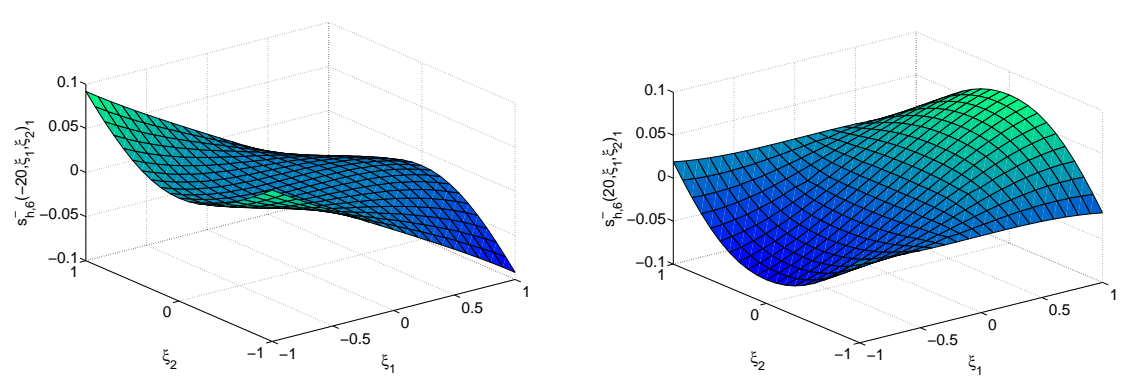

Fig. 13 Graphs of $s_{h, 6}^{-}\left(\tau, \xi_{1}, \xi_{2}\right)_{1}$ for $\tau=-20,20$

\section{References}

1. E. Allgower and K. Georg. Numerical continuation methods. An introduction, Springer Series in Computational Mathematics 13. Springer, Berlin etc., 1990.

2. B. Aulbach, M. Rasmussen and S. Siegmund. Invariant manifolds as pullback attractors of nonautonomous difference equations. In Proceedings of the Eighth International Conference of Difference Equations and Application (Brno, Czech Republic, 2003), 23-37. Chapman \& Hall/CRC, Boca Raton, 2005.

3. W.-J. Beyn. On the numerical approximation of phase portraits near stationary points. SIAM Journal of Numerical Analysis, 24(5):1095-1112, 1987.

4. W.-J. Beyn and J. Lorenz. Center manifolds of dynamical systems under discretization. Numerical Functional Analysis and Optimization, 9:381-414, 1987.

5. W.-J. Beyn and W. Kleß. Numerical Taylor expansion of invariant manifolds in large dynamical systems. Numerische Mathematik, 80:1-38, 1998.

6. H. Broer, H. Osinga and G. Vegter. Algorithms of computing normally hyperbolic invariant manifolds. Zeitschrift für angewandte Mathematik und Physik, 48(3):480-534, 1997.

7. F.H. Clarke. Optimization and nonsmooth analysis, Classics in Applied Mathematics 5. SIAM, Philadelphia, PA, 1990.

8. V. Chepyzhov and M. Vishik. Attractors for Equations of Mathematical Physics, Colloquium Publications 49. American Mathematical Society, Providence, RI, 2001

9. M. Dellnitz, G. Froyland and O. Junge. The Algorithms behind GAIO - Set Oriented Numerical Methods for Dynamical Systems. In Ergodic Theory, Analysis, and Efficient Simulation of Dynamical Systems, 145-174, Springer 2001.

10. M. Dellnitz and A. Hohmann. A subdivision algorithm for the computation of unstable manifolds and global attractors. Numerische Mathematik, 75:293-317, 1997.

11. P. Deuflhard. Newton methods for nonlinear problems. Affine invariance and adaptive algorithms, Springer Series in Computational Mathematics 35. Springer, Berlin, 2004.

12. T. Eirola and J. Von Pfaler. Taylor expansions for invariant manifolds numerically. $\mathrm{Nu}$ merische Mathematik, 99(1):25-46, 2004.

13. M. Fuming and T. Küpper. Numerical calculation of invariant manifolds for maps. Numerical Linear Algebra and Applications, 1(2):141-150, 1994.

14. B. Garay. Discretization and some qualitative properties of ordinary differential equations about equilibria. Acta Mathematica Universitatis Comenianae, LXII:249-275, 1993.

15. J. Guckenheimer and A. Vladimirsky. A fast method for approximating invariant manifolds. SIAM Journal of Applied Dynamical Systems, 3(3):232-260, 2004.

16. M.E. Henderson. Computing invariant manifolds by integrating fat trajectories. SIAM Journal of Applied Dynamical Systems, 4(4):832-882, 2005.

17. A.J. Homburg, H.M. Osinga and G. Vegter. On the computation of invariant manifolds of fixed points. Zeitschrift für angewandte Mathematik und Physik, 46(2):171-187, 1995.

18. M.S. Jolly and R. Rosa. Computation of non-smooth local centre manifolds. IMA Journal of Numerical Analysis, 25:698-725, 2005.

19. D. Jones and A. Stuart. Attractive invariant manifolds under approximation. Inertial manifolds. Journal of Differential Equations, 123:588-637, 1995.

20. M. Kanat Camlibel, J.-S. Pang and J. Shen. Conewise linear systems: Non-zeroness and observabiliy. SIAM Journal of Control and Optimization, 45(5):1769-1800,2006. 
21. S. Keller and C. Pötzsche. Integral manifolds under explicit variable time-step discretization. J. Difference Equ. Appl., 12(3-4):321-342, 2005.

22. C.T. Kelley. Solving nonlinear equations with Newton's method, Fundamentals of Algorithms 1. SIAM, Philadelphia, PA, 2003.

23. N. Koksch and S. Siegmund, Pullback attracting inertial manifolds for nonautonomous dynamical systems, Journal of Dynamics and Differential Equations 14 (2002), 889-941.

24. B. Krauskopf, H. M. Osinga, E. J. Doedel, M. E. Henderson, J. Guckenheimer, A Vladimirsky, M. Dellnitz and O. Junge. A survey of methods for computing (un)stable manifolds of vector fields. Int. Journal of Bifurcation and Chaos, 15(3):763-791, 2005.

25. R.F. Costantino, J.M. Cushing, B. Dennis and R.A. Desharnais, Experimentally Induced Transitions in the Dynamic Behavior of Insect Populations, Nature 375:227-230, 1995.

26. Y. Kuang and J. Cushing. Global stability in a nonlinear difference-delay equation model of flour beetle population growth. J. Difference Equ. Appl., 2(1):31-37, 1996.

27. B. Kummer. Newton's method for non-differentiable functions. In e. a. J. Guddad, editor, Advances in mathematical optimization, Mathematical Research, pp. 114-125, Berlin, 1988. Akademie.

28. G. Maggio, M. di Bernardo and M. Kennedy. Nonsmooth bifurcations in a piecewise-linear model of the Colpitts oscillator. IEEE Transactions on Circuits and Systems, 47(8):1160$1177,2000$.

29. R. Mifflin. Semismooth and semiconvex functions in constrained optimization. SIAM Journal of Control and Optimization, 15:959-972, 1977.

30. G. Moore and E. Hubert. Algorithms for constructing stable manifolds of stationary solutions. IMA Journal of Numerical Analysis, 19:375-424, 1999.

31. J. Ombach. Computation of the local stable and unstable manifolds. Universitatis Iagellonicae Acta Mathematica, XXXII:129-136, 1995.

32. C. Pötzsche and S. Siegmund, $C^{m}$-smoothness of invariant fiber bundles, Topological Methods in Nonlinear Analysis, 24:107-146, 2004.

33. C. Pötzsche. Attractive invariant fiber bundles. Applicable Analysis, 86:687-722, 2007.

34. C. Pötzsche. Discrete inertial manifolds. Mathematische Nachrichten (to appear).

35. C. Pötzsche and M. Rasmussen. Taylor approximation of invariant fiber bundles. Nonlinear Analysis (TMA), 60(7):1303-1330, 2005.

36. L. Qi and D. Sun. A survey of some nonsmooth equations and smoothing Newton methods. In e. a. Eberhard, A., editor, Progress in Optimization, Applied Optimization 30, pp. 121146, Dordrecht, 1999. Kluwer.

37. J.C. Robinson. Computing inertial manifolds. Discrete and Continuous Dynamical Systems, 8(4):815-833, 2002.

38. G.R. Sell and Y. You, Dynamics of evolutionary equations, Applied Mathematical Sciences, vol. 143, Springer, Berlin etc., 2002.

39. C. Simó. On the numerical and analytical approximation of invariant manifolds. In D. Benest and C. Froeschlé, editors, Les Methodes Modernes de la Mechanique Céleste, pp. 285329. Coutelas, 1989.

40. H. Xu and X. Chang. Approximate Newton methods for nonsmooth equations. Journal of Optimization Theory and Applications, 93(2):373-394, 1997. 